\title{
TIME-RESOLVED SPECTROPHOTOMETRY OF
}

HZ HERCULIS AND DQ HERCULIS

\author{
Gary Alan Chanan \\ (Ph. D. thesis)
}

January 1978

Prepared for the U. S. Department of Energy under Contract W-7405-ENG-48

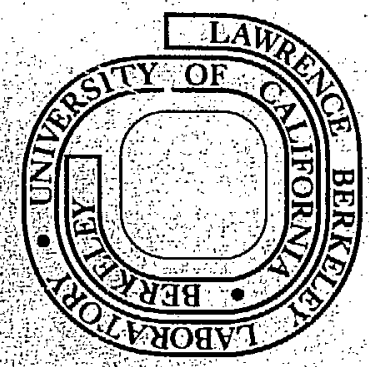




\section{LEGAL NOTICE}

This report was prepared as an account of work sponsored by the United States Government. Neither the United States nor the Department of Energy, nor any of their employees, nor any of their contractors, subcontractors, or their employees, makes any warranty, express or implied, or assumes any legal liability or responsibility for the accuracy, completeness or usefulness of any information, apparatus, product or process disclosed, or represents that its use would not infringe privately owned rights. 
ABSTI:16

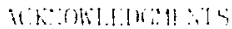

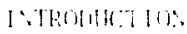

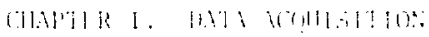

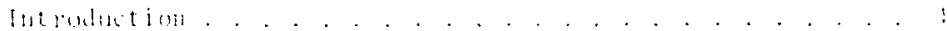

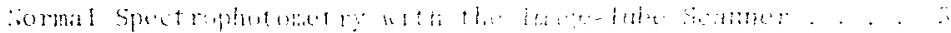

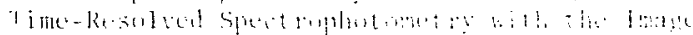

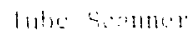

$\therefore+10$

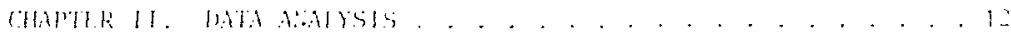

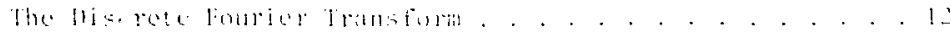

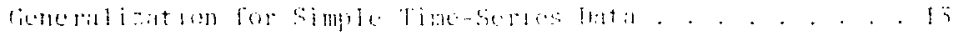

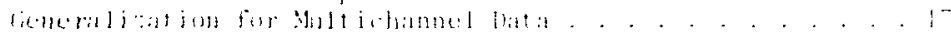

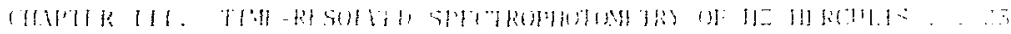

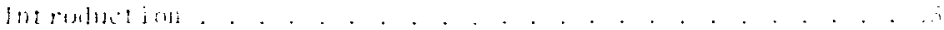

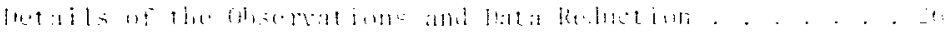

Re+ilis . . . . . . . . . . . . . . . . . .

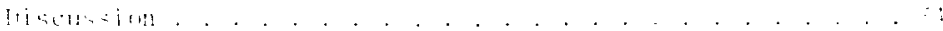

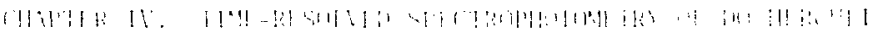

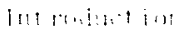

$[\ldots, 1: \cdots, \cdot, 1,1$

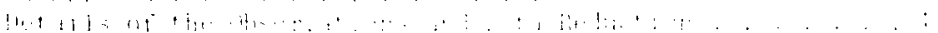

\{.

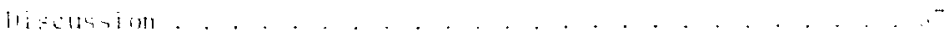

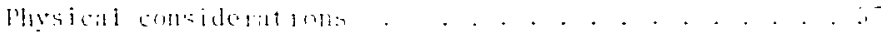

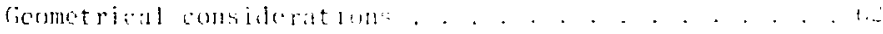

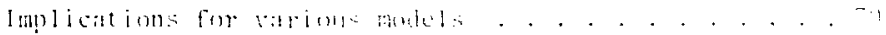

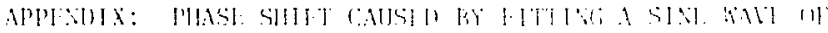

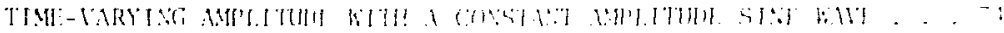

RIFFIRINCIES

F] CIJRE: CAITIONS

\section{FICURTS}

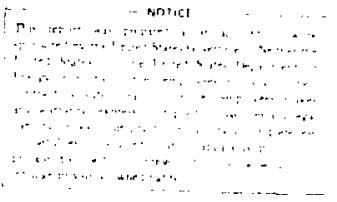


Man bedenke, welche Enthattsomkeit dazu gehört, sich so kurz zu fassen.

- Arnold Schoenberg 


\title{
TIME-RESOLVED SPECTROPHOTOMETRY OF \\ HZ HERCULIS AND DQ HERCULIS
}

\author{
Gary Alan Chanan \\ Lawrence Berkeley Laboratory \\ University of California \\ Berkeley, California 94720
}

Januaxy 1978

\begin{abstract}
With some simple modifications, the image-tube scanner at the $3 \mathrm{~m}$ telescope at Lick observatory can be used in a high time-resolution mode. We have employed this apparatus to study the spectral changes which occur during the pulse period in the optical pulsars $\mathrm{HZ}$ Herculis (1.2 $\mathrm{s}$ period) and DQ Herculis (71 s period). In this thesis we describe the data acquisition and develop the tools needed for the data analysis. We then present the results of the observations.

In the case of $\mathrm{HZ}$ Herculis (Hercules $X-1$ ), our observations cover the binary phase interval 0.18 to 0.26 and are concerned only with those pulsations that have been shown (Middleditch and Nelson, 1976) to criginate at the X-ray heated surface of the Roche lobe filling companion of the neutron star. We find that these pulsations are distributed throughout the optical continuum. Although the details of this distribution are not precisely determined, our observations appear to agree at least
\end{abstract}


qualitatively with the numerical results of other investigators. However, the data are not consistent with the stiggestion of Davidsen, Margon, and Middleditch (1975) that the pulsations result from the strong modulation of a small number of emission lines. Emission lines are seen, but are not strongly modulated $(<9 \%)$ and do not contribute significantly to the observed pu1sations.

The observations of $D Q$ Herculis cover one full binary cycle, excluding eclipse. We again find pulsations distributed throughout the continuum with generally weak wavelength dependence. However, in this case the emission line $\lambda 4686$ (He II) is more strongly modulated than the underlying continuum and exhibits an unexpected effect: The pulse phase increases rapidly with increasing wavelength across the line. This effect can be understood in terms of a simple model in which the pulsations arise at the inner edge of the accretion disk, excited by radiation which originates at hot spots o:: the white dwarf and which sweeps around the disk as the degenerate star rotates. A similar model in which the pulsations arise predominantly from the back half of the surface of the. disk, although more difficult to make quantitative, appears in several respects to be more promising. The evident relation between the phase shift across the emission line and the so-called $360^{\circ}$ phase shift through eclipse, discovered by Warner et al. (1972) is also discussed. 
ACKNOWLEDGMENTS

I am indebted to Tim Daly for preparing the figures used in this work, to Steven Hawley for reducing the scanner data on HZ Her, to Jonathan Katz for his critical reading of Chapter IV of the manuscript, to Bruce Margon for pointing out that $\mathrm{DQ}$ Her would be a good candidate for timeresolved spectrophotometry and for reducing the scanner data on this object, to Terry Mast and Richard Muller for many important and helpful conversations, to Joseph Miller for putting his telescope time and expertise at our disposal for the observations of $\mathrm{HZ} \mathrm{Her,} \mathrm{to} \mathrm{Mark} \mathrm{Roos} \mathrm{for} \mathrm{assistance}$ with the observations of DQ Her, and to John Saarloos for his invaluable technical assistance and for his enthusiasm.

I would like to thank my thesis committee - Eugene Commins, Christopher McKee, and Frank Shu - for reading the manuscript and for many useful discussions. I am particularly grateful to Eugene Commins for serving as my thesis adviser and for his time and encouragement over the last three years, to John Middleditch for carefully explaining his and Jerry Nelson's earlier work (on which much of this work is based) and for a great deal of additional help, and to Jerry Nelson for his patience and assistance throughout, and for giving me the opportunity to work on this project.

This work was done under the auspices of the U.S. Department of Energy. 


\section{INTRODUCTION}

The binary stars HZ Herculis and DQ Herculis are of considerable astrophysical interest for a variety of reasons (to be described below). of greatest concern here, however, is the fact that they are two of only a small number of stars known to exhibit rapid, stable optical pulsations. The mechanisms by which these stars pulsate, although presumably related to stellar rotation in one and possibly both cases, are not precisely known, and in fact are matters of some controversy (as we shall discuss). The main motivation for the present work is that we can hope to gain some insight into the pulsation mechanisms in these two objects by studying in some detail the spectral changes which occur during the pulse period.

To accomplish this purpose we need to monitor the star of interest in a large number (say tens or hundreds) of separate wavelength channels. However, since both stars are rather faint (about 14th magnitude) and the pulsations much fainter still, any simple scheme in which the wavelength channels are monitored in sequence (as, for example, with a series of filters], is impractical: We cannot afford to lose the photons arriving in a11 other channels while we are counting those arriving in one particular channel. In fact, the overall counting efficiency in such schemes is inversely proportional to the widths of the individual channe1s, and even for moderate wavelength resolution very low efficiency resul:s. Fortunately, an elegant solution to this problem is provided by the image tube scanner (ITS) at the $3 \mathrm{~m}$ telescope at Lick Observatory. This device, developed by J. Wanmler and L. Robinson of the Observatory, enables one 
to monitor up to 2048 wavelength channels simultaneously, without missing any photons in the process. Besides the enormous gain in efficiency, the simultaneity insures that any irregular variability in the star or in the observing conditions is not incorrectly interpreted as different behavior in different wavelength channels (as can happen with sequential monitoring). Finally, the time-resolution required for ouiz observations is an intrinsic, though not normally used, property of the ITS. In Chapter I we describe this device and the modifications necessary for our particular observations.

The signals from the two optical pulsars that we are concerned with are strictly periodic, so the discrete Fourier transform (DFT) is the basic tool used in our data analysis. We find it useful, however, to push the frequency resolution of the DFT beyond the nominal value of $\mathrm{T}^{-1}$ (where $\mathrm{T}$ is the total observing time). A method for doing this is described in the first part of Chapter II. Once we modify the DFT in this way, the phase information contained in the Fourier coefficients becomes transparent and turns out to be quite useful when applied to the observations.

The error analysis for our observations is made somewhat more complicated than in the usual power spectrum analysis because we need to take into account possible correlations in the noise from wavelength channel to wavelength channel, in addition to the more familiar (uncorrelated) noise due to counting statistics. Such correlations in fact turn out to be important for the observations of DQ Her, though not for the higher frequency $\mathrm{HZ}$ Her. In the second part of Chapter II we develop a scheme for separating and evaluating these two distinct noise contributions. This separation is statistical in nature and is based on the fact that 
there is a large number of wavelength channels.

In Chapter III we develop the necessary background material for the observations of $\mathrm{HZ} \mathrm{Her,} \mathrm{provide} \mathrm{the} \mathrm{details} \mathrm{of} \mathrm{the} \mathrm{observations,} \mathrm{and}$ describe the results and conclusions. Except for the data acquisition and analysis described in Chapters $I$ and $I I$, this chapter is self-contained. A sịmilar format is followed in Chapter IV for the observations of DQ Her. Chapter III does not differ substantially in content from the paper, "Measurement of the Spectrum of Optical Pulsations from HZ Herculis/ Hercules X-1," by J. E. Ne1son, G. A. Chanan, and J. Midd1editch (Ap. J. 212, 215). Chapter IV is based on the paper, "Time-Resolved Spectrophotometry of DQ Herculis: A Wavelength-Dependent Phase Shift in He II $\lambda 4686, "$ by G. A. Chanan, J. E. Nelson, and B. Margon (submitted to Ap. J.) . The greater length of Chapter IV reflects the fact that the interpretacion of the observations of DQ Her is more complicated than is the case for $\mathrm{HZ}$ Her, and in this chapter some of the points in the corresponding paper have been elaborated upon. The above papers have not been otherwise referenced in this work.

In the Appendix we examine briefly the effects that amplitude modulation can have on the measured phase of a periodic signal. Although this issue is not direct1y related to our own data analysis, it arises in the context of other, closely related observations of DQ Her. 
CHAPTER I. DATA ACQUISITION

Introduction

The image-tube scanner (ITS) (Robinson and Wampler, 1972) at the Lick Observatory $3 \mathrm{~m}$ telescope is a sensitiv, and linear detector which enables one to count photons in a large number (2048) of individual wavelength channe1s. The uniqueness and importance of the ITS stems from the fact that it does not suffer from either of two major drawbacks, one or the other of which plagues multichannel devices in general. The dilemma in such devices is that usually one must either: i) build, maintain, and calibrate individually a very large number of detectors, or ii) somehow sweep through all the channels with a single detector. The latter approach avoids the great expense and cumbersomeness of the former, but at the cost of a tremendous loss in sensitivity - all channels other than the one being monitored at a given time are effectively dead. The elegant solution of the ITS to the above problem is to use a phosphor screen as a short-term storage element. The wavelength channels are then scanned in sequence by a single detector, but since the phosphor decay time is not short compared to the scan cycle time (the time required to sweep the detector once through all channels) the detector counts effectively simultaneously in all channels and no photons are lost.

In the next section we will describe in some detail how the ITS works. This will be followed by a description of the way in which the ITS was modified for our own particular observations. 
Normal Spectrophotometry with the Image-Tube Scanner

Let us first outline the operation of the ITS; we will then go back and fill in the details. A block diagram illustrating the scanner operation is shown in Fig. I-1. The image (spectrum) from a diffraction spectrograph fa1ls on the photocathode of the first of a chain of three image tubes and is reproduced with an amplification of $\sim 10^{5}$ on the phosphor of the final tube. The image persists here for a period of several milliseconds, due to the finite decay time of the phosphor. In a period of time comparable to the phosphor decay, the image is scanned by a magnetically controlled image dissec sor. Due to the combined effects of the amplification and persistence of the phosphor, the probability of detecting one or more photons from the phosphor for each photoelectron produced at the original photocathode is high, despite the fact that each element of the image is only examined for a very small fraction of the total counting time. Pulses (corresponding to individual detected photons) from the output of the dissector trigger a discriminator-amplifier and are then counted in a circulating memory. At the end of the total integration period (typically $10^{4}$ to $10^{6}$ cycles), the memory is read out onto magnetic tape. The scanner operation is controlled by a PDP 8/I computer.

In one cycle the scanner sweeps through 4096 individual channels, each one corresponding to a different physical point on the image. This image normally consists of two spectra (2048 channels each) - one of the star of interest and one of the sky alone, so that the (often substantial) background can be subtracted. The scanner dwells for $1 \mu$ in each channel; 
the stepping rate is controlled by a $1 \mathrm{MHz}$ crystal oscillator. During each 1 us dwe11, individual pulses from the dissector are counted in a 4-bit scaler and, near the end of this interval, these counts are added into the 24-bit, 4096-word circulating memory. There is a period of 150 us of dead time after each scan to allow for the fly-back of the scan sweeps. The time for the entire scan cycle of star plus sky spectra is thus about $4.4 \mathrm{~ms}$.

In order to enhance the probability of counting a photon from the phosphor given a photoelectron emitted from the photocathode, the scanner is adjusted to have a high photon counting multiplicity. That is, each photoelectron ultimately produces $\sim 7$ pulses at the output of the dissector. Clearly this multiplicity affects the photon counting statistics; this fact is taken into account in the statistical analysis of Chapter II.

For typical moderate wavelength resolution spectrograph parameters, each channel of the ITS is $\sim 1.2 \AA$ in width and the channels are contiguous, so that a spectral range of about $2500 \AA$ is covered. The ITS is somewhat over-resolved in this mode - the intrinsic wavelength resolution depends on the width of the spectrograph slit but is typically several Angstroms and hence several channels. The (non-linear) relation between wavelength, $\lambda$, and channel number, $k$, (the latter corresponding to position on the phosphor screen) is not calculated in advance. It is determined by recording spectra of emission line lamps and fitting $\lambda$ versus $k$ for the known emission features with a polynomial of sufficiently high degree usually a cubic - so that the resulting wavelength residuals are acceptably small. The system response is calibrated by recording spectra of well-known, usually reatureless, standard stars. 
The main problem with the ITS is that, after exposure to a given light level, the phosphor glows (with a nonexponential tail) at a fraction of a percent of that level for a very long time $(\sim 1 / 2$ hour). In practice this dr. ; not create any serious difficulties, except that care must be taken to plan the observing program so that faint objects are not observed soon after bright ones.

The practical dynamic range of the ITS is quite wide - objects from tenth to twenty-second magnitude can be studied. The faint limit is set by the dark current (which is now comparable to the brightness of one square arc second of the Mt. Hamilton night sky and thus cannot profitably be reduced further). The bright limit is established by the abovementioned afterglow problem. The linearity of the system is very good; standard stars of tenth magnitude are used routinely to calibrate the faintest sources. When used as a photometer, the ITS is accurate to about $10 \%$. The sky subtraction is satisfactory. Cancellation of the emission lines in the night sky $(0$ I $\lambda 5577$ and severa1 lines of $\mathrm{Hg}$ I mostly due to scattering from sources in the city of San Jose) can be achieved to about $1 \%$ accuracy. These features are not visible at all, for example, in the sky-subtracted spectrum of DQ Her (Fig. IV-1), whereas they were of about the same intensity as the most proilinent stellar features in the raw (unsubtracted) spectrum. Of course there is an increase in the noise level which cannot be avoided in the wavelength channels corresponding to these sky features.

Further details of the operation of the ITS can be found in the article by Robinson and Wampler (1972) cited above. 
Time Resolved Spectrophotometry with the Image-Tube Scanner

In nomnal spectrophotometry the numbers of incoming photons are recorded as a function of wavelength; we will define time-resolved spectrophotometry here as a process in which the numbers of incoming photons are recorded as a function of wavelength and time. In this section we will describe the modifications to the ITS that are required in order to use it in a time-resolved mode; i.e., for time-resolved spectrophotometry. Since, as described above, the ITS is an intrinsically time-resolved device (the scan cycle period provides an internal clock), the necessary modifications are not extensive. However, two important factors make the task nontrivial: i) the scan cycle period does not provide a time base that is stable enough for our purposes, and ii) huge amounts of data are involved.

The variability of the scanner time base is due to the fact that the dead time for the flyback hetween scans is not controlled by the crystal oscillator, but rather by a $150 \mu$ s one-shot (see Fig. I-1). The period is thus essentially set by an RC decay time. We have adapted our high-speed data recording system, normally used for simple fast photometry (Middleditch, 1975; Nelson, Chanan, and Peterson, 1978) to get around this problem - we provided a stable and accurate external time base of $10 \mathrm{~ms}(100 \mathrm{~Hz})$ as described in the above papers and then considered only a single ITS scan of the star within each $10 \mathrm{~ms}$ interval. The recording system was shut down from the arrival of a given $10 \mathrm{~ms}$ clock pulse until the beginning of the next full ITS star scan as signalled by the rising (or falling) edge of the gate in Fig. I-1. Pulses from the 
output of the discriminator-amplifier were then counted in one of two 4-bit scalers for 256 contiguous periods of 8 us each, as timed by the $1 \mathrm{MHz}$ ITS crystal oscillator. At the conclusion of the even-numbered 8 us intervals, the contents of the two 4-bit scalers were packed into an 8-bit word and sent to a Kennedy 8232 buffered formatter. From here they were written periodically onto nine-track magnetic tape with a Kennedy 9000 synchronous tape transport.

The net effect of the above recording schere was to add (passively) 8 scanner channels together at a time so that a 256-point spectrum (each point or channel corresponding to about $10 \AA$ in width) was recorded every $10 \mathrm{~ms}$. Since the first word of each such spectrum also included tube noise from between scans, only the remaining 255 points will be considered from here on.

The scanner channels were added together both to cut down the data rate (we ran at close to the limiting rate of the Kennedy recording system) and to render the subsequent data analysis more tractable. The wavelength resolution of the observations to be described in succeeding chapters is determined, for the most part, by the intrinsic resolution of the scanner, not by the number of scanner channels summed together in the above process. By recording only one scan every $10 \mathrm{~ms}$ according to the above scheme, we not only avoided the timing instability of the ITS; the data rate was cut further by more than a factor of two, but with little or no loss of information, due to the long decay time of the phosphor. Even with these measures, the amount of data collected was enormous. The observations of $\mathrm{HZ} \mathrm{Her,} \mathrm{for} \mathrm{example,} \mathrm{involved} \mathrm{about} 10^{9}$ bits of information and took up several miles of magnetic tape. 
The interface between the scanner electronics of Fig. I-1 and our data-recording system was essentially contained in two electronic modules: i) a nine-track tape interface (13V-6950) normally used in our fast photometry system, and ii) a supplemental control box (14X-4530-51). These modules will not be described in further detail here; schematic diagrams of both are on file at the Lawrence Berkeley Laboratory. The recording system was connected to the ITS in parallel with the electronics of Fig. I 1, so that the normal operation of the scanner was not interfered with during our observations. Traditional 2048-channel sky-subtracted spectra with long ( $\sim 20$ minute) integration times were thus recorded during a11 of our time-resolved observations.

Because of the very high-speed capability of the time-resolved system, we used it to observe briefly the we11-known optical pulsar in the Crab nebula. However, due to the fact that the phosphor decay time and the scan cycle time are not negligible compared to the very short ( $\sim 33 \mathrm{~ms}$ ) pulse period of this object, it is difficult to interpret these data and they will not be discussed in this work. These observations were useful, though, in establishing that the time-resolved system worked properly and in verifying that the phosphor decay time was of order $10 \mathrm{~ms}$ and thus inconsequential on the much longer $(\gtrsim 1$ s) timescales dealt with in the observations described in succeeding chapters.

Note

In the time since the observations described below were made, the ITS has been modified so that, except for very high frequency observations, much of the time-resolved data recording scheme described above is rendered 
effectively obsolete. These nodifications are: i) the flyback dead time is now clocked precisely $(128 \mu \mathrm{s})$ by the $1 \mathrm{MHz}$ oscillator, and ii) the capacity of the PDP $8 / 1$ which rums the ITS has been enlarged sustantially. The first modification eliminates the timing instability due to the oneshot. The second modification enables the computer to record successive short-integration ( $\geqslant 0.1 \mathrm{~s})$ spectra (and have them written on magnetic tape) without interfering with its normal task of controlling the scanner operations. 
CHAPTER II. DATA ANALYSIS

\section{The Discrete Fourier Trans form}

Let us consider a time-series of data, $d_{j}(j=0,1, \ldots, N-1)$, in our case the numbers of photons arriving in $\mathrm{N}$ contiguous time bins each of duration $\tau$. The discrete Fourier transform (DFT), denoted $A(\ell)$, is defined for $\ell=0,1, \ldots, \mathrm{N}-1$ as

$$
A(\ell)=\sum_{j=0}^{N-1} d_{j} e^{-2 \pi i j \ell / N}
$$

(see, for example, Brigham, 1974). The DFT defined in this way has a number of useful properties (described in detail in the above reference). Perhaps the most important of these is the existence of a machine computation algorithm (Cooley and Tukey, 1965) which reduces the number of computer operations needed to perform the transform from $\mathrm{N}^{2}$ to $2 \mathrm{~N} \log _{2} \mathrm{~N}$ when $\mathrm{N}$ is an integral power of 2 , and results in an enormous savings of time and money for large $N$.

We will not duplicate the usual analysis of the DFT here. However, we have found it useful to generalize the DFT, and this will require some discussion. There are two important motivations for this generalization: i) the frequency resolution of the DFT can be improved substantially beyond the nominal value of $\sim \mathrm{T}^{-1}$, where $\mathrm{T}=\mathrm{N} \tau$ is the total observing time, and $i$ i) it is difficult to extract phase information from the DFT of Eq. (II.1). These two matters are clearly related; if the frequency of the signal is known only to within $\Delta f= \pm(2 \mathrm{~T})^{-1}$, then the corresponding uncertainty in the signal phase is $\Delta \phi=2 \pi \Delta \mathrm{fT}= \pm \pi$. We will describe 
effectively obsolete. These modifications are: i) the flyback dead time is now clocked precisely $(128 \mu \mathrm{s})$ by the $1 \mathrm{MHz}$ osrillator, and ii) the capacity of the PDP $8 / I$ which runs the ITS has been enlarged substantially. The first modification eliminates the timing instability due to the oneshot. The second modification enables the computer to record successive short-integration $(\geqslant 0.15)$ spectra (and have them written on magnetic tape) without interfering with its normal task of controlling the scanner operations. 
CHAPTER II. DATA ANALYSIS

The Discrete Fourier Transform

Let us consider a time-series of data, $d_{j}(j=0,1, \ldots, N-1)$, in our case the numbers of photons arriving in $\mathrm{N}$ contiguous time bins each of duration $\tau$. The discrete Fourier transform (DFT), denoted $A(\ell)$, is defined for $\ell=0,1, \ldots, \mathrm{N}-1$ as

$$
A(\ell)=\sum_{j=0}^{N-1} d_{j} e^{-2 \pi i j \ell / N}
$$

(see, for example, Brigham, 1974). The DFT defined in this way has a number of useful properties (described in detail in the above reference). Perhaps the most important of these is the existence of a machine computation algorithm (Cooley and Tukey, 1965) which reduces the number of computer operations needed to perform the transform from $\mathrm{N}^{2}$ to $2 \mathrm{~N} \log _{2} \mathrm{~N}$ when $\mathrm{N}$ is an integral power of 2 , and results in an enormous savings of time and money for large $N$.

We will not duplicate the usual analysis of the DFT here. However, we have found it useful to generalize the DFT, and this will require some discussion. There are two important motivations for this generalization: i) the frequency resolution of the DFT can be improved substantially beyond the nominal value of $\sim T^{-1}$, where $T=N T$ is the total observing time, and ii) it is difficult to extract phase information from the DFT of Eq. (II.1). These two matters are clearly related; if the frequency of the signal is known only to within $\Delta f= \pm(2 T)^{-1}$, then the corresponding uncertainty in the signal phase is $\Delta \phi=2 \pi \Delta \mathrm{fT}= \pm \pi$. We will describe 
the appropriate generalization of the DFT below, first for a simple tineseries and then for multichannel data. The multichannel analysis is not a trivial extension of the procedure for a simple time-series because one needs to take into account the possibility of channel-to-channel correlations in the noise.

\section{Generalization for Simple Time-Series Data}

For an arbitrary angular frequency $\omega$ we define $A(\omega)$ by

$$
A(\omega)=\sum_{j=0}^{N-1}\left(d_{j}-\bar{d}\right) e^{-i j \omega \tau}
$$

where $\bar{d}$ denotes the average of the $\dot{d}_{j}$. Although the relation between this definition and the DFT of Eq. (II.1) is evident, we shall not attempt to motivate the choice of the precise form of Eq. (II.2) here in any detail. In the following we shall only be interested in this function in a neighborhood of a single frequency, which we shall denote by $\omega_{0}$. Now let us suppose that the data $d_{j}$ describe a sine wave of angular frequency $\omega_{0}$, i.e., $d_{j}=s_{j}$, where (writing $t_{j}$ for $j \tau$ )

$$
s_{j}=\alpha_{0} \cos \left(\omega_{0} t_{j}+\phi_{0}\right)
$$

subject to the restrictions

$$
\begin{gathered}
\omega_{0} T \ll 1 \\
N \omega_{0} T \gg 1
\end{gathered}
$$

These conditions simply require that the sampling frequency is high compared to the frequency of interest, and that the observations span a large number of cycles of the signal. Under the conditions (II.4) it 
is easy to show that

$$
\mathrm{A}\left(\omega_{0}\right) \approx 1_{2} N \alpha_{0} e^{i \phi_{0}}
$$

and the signal amplitude and phase may be determined directly from $A\left(\omega_{0}\right)$, provided that $\omega_{0}$ is known in advance. (The extent to which the relation [II.5] departs from equality is not of direct concern to us here; it is only meant to he suggestive. The actual uncertainties involved in the situation of interest will be considered explicitly below.)

In general, of course, we do not know $\omega_{0}$ in advance; we only have an approximate idea from the location of the spikes in the power spectrum (the absolute value squared of the DFT). Furthermore, the signal is always superimposed upon (and frequently dominated by) a background of noise counts. To be specific let us consider the case:

$$
d_{j}=s_{j}+n_{j}
$$

with the $s_{j}$ as in Eq. (II.3) and where the $n_{j}$ are noise counts given by a Poisson distribution with standard deviation $\sigma$. In addition to the assumptions (II.4), we shall assume that the signal-to-noise ratio in the individual time bins in small in the sense:

$$
\frac{\alpha_{0}}{\sigma} \ll N^{\frac{1}{2}} \omega_{0} \tau
$$

Under these conditions we can show that, in the vicinity of a spike in the power spectrum, the best estimate for the signal angular frequency $\omega_{0}$ is given by that value of $\omega$ (call it $\omega_{1}$ ) which maximizes $|A(\omega)|$, with $A(\omega)$ as in Eq. (II.2). The best estimates for the signal amplitude and phase, $\alpha_{1}$ and $\phi_{1}$ respectively, are given, as suggested by the relation 
(II.5), by

$$
A\left(\omega_{1}\right)=I_{2} N \alpha_{1} e^{i \phi_{1}}
$$

These estimates of the signal parameters are the best in the sense of fitting the data by minimizing $x^{2}$ as a function of the signal frequency, amplitude, and phase: It is straightforward (though tedious) to show that the above values for $\alpha_{1}, \phi_{1}$, and $w_{1}$ differ from those which actually minimize $\chi^{2}(\alpha, \phi, \omega)$ by terms which, under the above conditions, are shra11 compared to the statistical uncertainties in these quantities, the latter to be derived in the following. Note that while $\chi^{2}$ must be minimized with respect to three parameters, the corresponding quantities in the above procedure can be deduced by maxinizing a function of only one parameter.

It will be convenient at this point to separate $A(\omega)$ into two parts, $A_{s}(w)$ and $A_{n}(w)$, due to signal and noise respectively. By the linearity of $\mathrm{Eq}$. (II.2) we have, for any angular frequency $w$ :

$$
A(\omega)=A_{s}(\omega)+A_{n}(\omega)
$$

where

$$
\begin{aligned}
& A_{s}(\omega)=\sum_{j=0}^{N-1}\left(s_{j}-\bar{s}\right) e^{-i \omega t_{j}} \\
& A_{n}(\omega)=\sum_{j=0}^{N-1}\left(n_{j}-\bar{n}\right) e^{-i \omega t_{j}}
\end{aligned}
$$

Under the conditions (II.4) and (II.7), the dominant source of error in the determination of the signal parameters from $A\left(\omega_{2}\right)$ is the contribution to $A\left(w_{1}\right)$ of the term $A_{n}\left(w_{1}\right)$. We therefore need to investigate the statistical behavior of this term. 
By treating the sum in Eq. (II.10) as a random walk in the complex plane, one can show that the real and imaginary parts of $A_{n}(\omega)$ (for any $\omega$ ) Given by two identical Gaussian distributions, independent of frequency, centered about the origin. Let us define the power, $P(\omega)$, at a given angular frequency and the expectation value of the noise power $\bar{p}(\omega)$ as

$$
\begin{aligned}
& P(\omega)=|A(\omega)|^{2} \\
& \left.\bar{p}(\omega)=\left|A_{n}(\omega)\right|^{2}\right\rangle
\end{aligned}
$$

where the brackets denote ensemble averaging over the noise. From Parseval's theorem (c.f. Brigham, 1974) and the frequency independence of the distributions of the real and imaginary parts of $A_{n}(\omega)$, it follows immediately that $\overline{\mathrm{p}}=\mathrm{N} \sigma^{2}$, independent of $\omega$. However, in the case (frequent1y encountered in practice) of non-ideal statistics, the expectation value of the noise power may no longer be independent of frequency. We can still salvage the analysis, though, by replacing the theoretical value for $\bar{p}$ with an empirically determined local average (averaging over frequencies close to, but excluding, the frequency of interest).

We define the normalized Fourier transform $A^{\prime}(\omega)$ as:

$$
A^{\prime}(\omega)=A(\omega) \bar{p}^{-\frac{1}{2}}
$$

and similarly for $A_{S}^{\prime}(\omega)$ and $A_{n}^{\prime}(\omega)$. The Gaussian distributions of the real and imaginary parts of $A_{n}^{\prime}(\omega)$ then have mean squared deviations of $\frac{1}{2}$, and, in the absence of a signal $\left(\alpha_{0}=0\right)$ the power is distributed with an exponential probabịlity, P.:

$$
P(p / \bar{p}) d(p / \bar{p})=e^{-(p / \bar{p})} d(p / \bar{p})
$$


From the above argument and the linearity of the Fourier transform it follows directly that the uncertainties $\delta \alpha$ and $\delta \phi$ in the amplitude and phase of a signa1 determined according to the above procedure are given by:

$$
\begin{aligned}
& (\delta \alpha)^{2}=\frac{\bar{p} \alpha_{1}^{2}}{2 p\left(\omega_{1}\right)} \\
& (\delta \phi)^{2}=\frac{\bar{p}}{2 p\left(\omega_{1}\right)}
\end{aligned}
$$

for $p\left(\omega_{1}\right) / \bar{p}$ sufficiently large. The uncertainty $\delta \omega$ in the angular frequency is more difficult to determine, but follows from the equivalent procedure of minimizing $\chi^{2}$ :

$$
(\delta \omega)^{2}=\frac{6 \bar{p}}{p\left(\omega_{1}\right) T^{2}}
$$

where $\mathrm{T}=\mathrm{N} \tau$.

\section{Generalization for Multichannel Data}

Let us now consider our multichannel data, in particular the set of complex numbers $A_{k}(\omega)$, for a given angular frequency $\omega$, where $k=1,2, \ldots, 255$ is the wavelength channel number. The $A_{k}(\omega)$ are calcuIated from Eq. $\left(\right.$ II.6) with $\left(d_{j}-\bar{d}\right)$ replaced by $\left(d_{j k}-\bar{d}_{k}\right)$, where the $d_{j k}$ are the time series data in the $k^{\text {th }}$ wavelength channel. For want of a better term, we shall refer to these numbers, for a given angular frequency, as the Fourier coefficients at that frequency. By the linearity of the Fourier transform, the coefficient $A(\omega)$ for the broadband data $d_{j}$ 
(defined as the sur over $k$ of the $d_{j k}$ ) is related to the individual coefficients $A_{k}(\omega)$ by

$$
A(\omega)=\sum_{k} A_{k}(\omega)
$$

In all of our subsequent analysis we will determine the frequency of interest by maximizing $A(\omega)$ (as described in the previous section) for the broadband data, not by determining the best frequency for each individual wavelength channel. This procedure could conceivably result in some loss of information - one could imagine different wavelengths being pulsed at different frequencies - but we shall not consider that passibility here. The procedure does result, however, in a substantial reduction of noise in the individual wavelength channels. There is one additional matter of convention. When dealing with a particular frequency, we will not, in general, be interested in the absolute phase of the signal. It will be convenient, then, to subtract the broadband signal phase from the phases in all the individual wavelength channels. According to this convention the broadband signal phase will be zero, and the broadband coefficient $A(\omega)$ will be real and positive.

Clearly now, the amplitude and phase of the signal in each individual wavelength channel are determined from the $A_{k}(\omega)$ using Eq. (II.8), exactly analogous to the single-channel case discussed in the previous section. A11 that remains is to determine the appropriate normalization of the $A_{k}(\omega)$ and hence the uncertainties associated with the above individual amp1itudes and phasès.

The determination of errors (or noise) is, at least in the case of non-ideal statistics, somewhat complicated. In the case of the noise 
determination for single-channel data, non-ideal statistics can be dealt with simply, as described in the preceding section, by replacing the theoretical value for the (ensemble) average noise power with an empirical average over nearby frequencies. However, for multichannel data the corresponding procedure does not work in general. The problem is that one needs to take explicit account of possible correlations in the noise between one wavelength channel and another. To make this point clearer it will be convenient, in the following discussion, to divide sources of noise into two types, I and II, according to whether the noise is uncorrelated or correlated, respectively, from channel to channel. To enable us to discuss noise in general in a quantitative manner, we will define the noise in any wavelength channel and at any frequency as the measured Fourier coefficient for the corresponding wavelength and frequency when there is no a priori reason to expect a signal at that frequency.

Type I noise includes contributions from counting statistics and, in our case, possibly unknown sources of noise inherent in the ITS as well. To the extent that type I noise is dominated by counting statistics, this noise should be proportional to the square root of the number of photons counted in any particular wavelength channel; however; we shall not find it necessary to assume this $\sqrt{n}$ behavior in the procedure described below. Type II noise, on the other hand, is dominated by effects like fluctuations in the telescope guiding, sky transparency, and seeing, which are strongly (we shall assume $100 \%$ ) correlated from channel to channel, since the wavelength dependence of such effects is in general weak or non-existent. Because the net effect of such sources of type II noise is to prevent some fraction of photons (independent of wavelength) from being counted 
in a given time interval, we sha11 assume that this noise is directly proportional to the number of photons counted in any given channel.

To distinguish between and separately measure these two types of noise, we have devised the following procedure: For an arbitrary frequency in the range of interest, we calculate the entire set of 255 complex Fourier coefficients (one coefficient for each wavelength channn1) and repeat the calculation for each of a large number $\mathrm{N}_{f}$ of independent frequencies nearby but excluding the frequency of interest. Each set of 255 coefficients is then plotted in the complex plane, with the real and imaginary axes rotated so that the broadband coefficient at the given frequency lies along the positive real axis. An example of such an offfrequency plot is shown for the DQ Her data in Fig. IV-3a (we can ignore the normalization of the coefficients in this plot for the moment). Now, since type II noise is assumed to affect all coefficients at a given frequency in the same way, it will cause a displacement of all the coefficients in the same direction (which must therefore be the direction of the broadband coefficient, i.e. the real axis), and this displacement will be proportional to the number of photons counted in the wavelength channel associated with a given coefficient. It follows that the imaginary parts of the Fourier coefficients are virtually unaffected by sources of type II noise. The rms imaginary part of the Fourier coefficient in any given wavelength channe1, averaged over the $\mathrm{N}_{\mathrm{f}}$ frequencies, thus gives a relatively pure measure of the type I or uncorrelated noise in that channel. Specifically, denoting the rms imaginary part of the coefficient for the $k^{\text {th }}$ channel by $I_{k}$, we shall normalize the 255 coefficients according to: 


$$
A_{k}^{\prime}(\omega)=\frac{A_{k}(\omega)}{I_{k} \sqrt{2}}
$$

analogous to Eq. (II.12). All the scatter plots in Figs. III-2 and IV-3 have been normalized in this way; the uncertainties associated with type I noise in the real and imaginary of the coefficients in these figures are given by $\pm 1 / \sqrt{2}$. Note that nowhere in this normalization procedure is it assumed that type $I$ noise is defined by $\sqrt{n}$ statistics. This is particularly important for the observations under discussion in this work as the (rather poorly known) value of the counting multiplicity of the ITS is automatically taken into account.

To find the contribution of type II noise at the frequency of interest, we analyze the broadband data (summed over wavelength) according to the single-channel scheme defined in the preceding section. Again the noise determination involves examining the fluctuations at frequencies nearby but excluding the frequency of interest. (This is particularly important here since type II noise is essentially indistinguishable from a broadband signal.) This broadband analysis, Eq. (II.16), and the assumption that type II noise scales with the number of photons counted, are sufficient to determine the type II noise contribution at the signal frequency. The errors in the various quantities derived from the Fourier coefficients are determined from the combined effects of type $I$ and type II noise. In fitting a series of points derived from these coefficients it must be remembered that type II noise affects all the coefficients in the same way. In general, the contribution due to the latter must therefore be folded in after the fit has been made. 
We can see the effects of type II noise directly in Fig. IV-3a, which is typi al of the DQ Her data. Upon close examination it is clear that the centroid of this distribution of coefficients has been displaced to the right. While this displacement is small, it would nonetheless be extremely improbable in the absence of channe1-to-channel correlations in the noise. In general, in the DQ Her data, type II noise contributions are small in the individual wavelength channels (although type II noise dominates in the broadband) and in the HZ Her data, type II noise is negligitle. We could not have predicted this situation in advance, although we would expect type II noise to become relatively more important at lower frequencies (i.e. $\sim 0.01 \mathrm{~Hz}$ ) than at higher frequencies (i.e. $\sim \mathrm{Hz}$ ). This is due to the fact that the characteristic time scales for the sources of type II noise - variability in telescope guiding, sky transparency, and seeing - tend to be rather long.

Loosely speaking, we can summarize the above discussion as follows: Type I noise (in the absence of type II noise) causes the characteristic $1 / \sqrt{2}$ scatter about the origin of the Fourier coefficients in plots like Figs. III-3a and IV-3a. Type II noise then displaces the whole distribution of coefficients in a given direction (evident in the latter figure but not in the former, in which type II noise is negligible). of course, in the usual broadband or single-channel analysis we are only concerned with a single coefficient and the distinction between type I and type II noise cannot be made. 
CHAPTER III. TIME-RESOLVED SPECTROPHOTOMETRY OF HZ HERCULIS

\section{Introduction}

The eclipsing binary $\mathrm{HZ}$ Herculis/Hercules $\mathrm{X}-1$ is unquestionably one of the most important stellar objects in astrophysics in the 1970s. This system contains a compact object - presumably a neutron star - that is a source of X-rays pulsed with a precisely defined period (1.238 s). This provides a clock which enables one to measure accurately the orbital velocity of the $X$-ray source $\left(169 \mathrm{~km} \mathrm{~s}^{-1}\right)$ and the projected radius of the orbit $\left(3.95 \times 10^{11} \mathrm{~cm}\right)$ (Tananbaum et al., i972). The normal companion star is of rather low intrinsic luminosity so that the effects of the $X$-rays impinging upon this star are easily observed (Davidsen et al., 1972; Forman, Jones, and Liller, 1972; Bahcall and Bahcall, 1972). Weak optical pulsations from the system were discovered by Davidsen et a2. (1972) and investigated further by Middleditch and Nelson (1973) and Groth (1974). Because the dimensions of the binary system are large. compared to the distance light travels in one pulse period, the optical. (and underlying $X$-ray) pulsations provide a useful geometrical probe of the system (Middleditch, 1975; Middleditch and Nelson, 1976 [hereafter MN]). This probe has been exploited by these latter authors to make an acrurate determination of the mass of the neutron star $\left(1.30 \pm 0.14 \mathrm{M}_{\theta}\right)$.

The behavior of $\mathrm{HZ}$ Her/Her $\mathrm{X}-1$ on a variety of timescales at both $\mathrm{X}-\mathrm{ray}$ and optical wavelengths is extraordinarily complicated and a: complete description is beyond the scope of this work. The $\mathrm{X}$-ray: behavior is discussed in some detail by Giacconi et at. (1973); the optical behavior - on long timescales compared to the pulse period - by 
Deeter et al. (1976). The optical pulsations have been studied and described in depth by MN. Those points of MN which are most important for the present work are summarized in the following three paragraphs. We sha11 find it convenient below to use the name "HZ Her" to refer to the normal star alone and "Her $X-1$ " to refer to the neutron star companion. The word "pulsations" unless otherwise noted will imply optical pulsations:

(j) The pulsations are sinusoidal, have periods near but not identical to the period of the $X$-ray pulsar, and have amplitudes corresponding to about $0.1-0.2 \%$ of the maximum visible 1 ight from the system (in contrast to the strong modulation of the $\mathrm{X}$-ray source [Tananbaum et al., 1972; Doxsey et al., 1973]). In MN and the present work the $\mathrm{X}$-ray pulse period is taken to be $1.2378175 \mathrm{~s}$ (Giacconi, 1975).

(ii) The pulsations are intermittent and strongly correlated with the binary phase of the system (binary perfod $=1.700165 \mathrm{~d}$ [Giacconi et al., 19731). They are shuwn to originate both at (or near) the Roche lobe filling surface of $\mathrm{Hz}$ Her (region I: binary phase $\phi \sim 0.25$, region II: $\phi \sim 0.75$ ) and near the neutron star companion (region III: $\phi \sim 0.85$ ). Only those pulsations associated with the surface of $\mathrm{HZ}$ Her will be dealt with in the present work.

(iii) Pulsations from the surface of $\mathrm{HZ}$ Her are the result of a reprocessing of the incident $X$-ray energy in the atmosphere of the star, but the broadband observations of MN do not provide any insight into the details of this reprocessing mechanism. Basko and Sunyaev (1973) have suggested that the pulsations may be due to pulsing emission lines which result from the recombination of $X$-ray ionized atoms in an optically thin region (see Crampton and Hutchings [1974] for a description of the 
[highly variable] emission features from this system). Detailed numerical models of the interaction of the X-rays with the atmosphere of $\mathrm{HZ} \mathrm{Her}$ have been constructed by Alme and Wilson (1974) and by Anderson (1977). A pulsing continuum is a general feature of these latter models, although pulsing emission lines are not ruled out.

The present work concerns the wavelength distribution of the optical pulsations, data which allows one, at least in principle, to distinguish between the various possible pulsation mechanisms. Previous experiments which addressed this question were carried out by Canizares and McClintock (1975), who searched for pulsations in the most prominent emission features, the N III - C III complex (centered near $4640 \AA$ ) and He II $\lambda 4686$, with negative results, ${ }^{1}$ and by Moffett, Nather, and Vanden Bout (1974) who obtained similar negative results in the $\lambda 4640$ complex alone. Interestingly, one run of Moffett et al. was simultaneous with run 86 of MN in which broadband pulsations were seen. However, Davidsen, Margon, and Middleditch (1975) (hereafter DMM), in more recent observations using a $100 \AA$ interference filter, have reported the detection of such pulsed emission lines (from region I). A total pulsed equivalent width of $\sim 2 \AA$ and a total pulsed fraction of greater than $25 \%$ was reported for the He II and N III lines combined. These features were further reported to be pulsing in phase with the broadband signal, and it was suggested that all of the optical pulsations may in fact be due to a

$1_{\text {The absence of pulsations reported by Canizares and McClintock may }}$ not be significant, since the binary phases during which pulsations are most often present (cf. MN) were not covered in their observations. 
small number of such pulsed emission lines.

Our results are in disagreement with the' reported detection of pulsed emission lines and with the resulting hypothesis of DMM. In addition, a search of the literature has established that on at least one occasion (JD 2441823.85 - 2441823.97) when relatively strong pulsations were present (run 46 of $\mathrm{MN}$ ), simultaneous observations (Bopp et al., 1973) showed no emission lines in the spectrum of $\mathrm{HZ}$ Her down to a limit of $0.25 \AA$ equivalent width. This provides independent evidence that the pulsations are not due to a small number of prominent emission features.

Details of the Observations and Data Reduction

Spectra of $\mathrm{HZ}$ Her were recorded for a 3-hour period on 1 October 1975 beginning at JD 2442686.615, covering the binary phase interval $0.18-0.26$. This night was selected because the optical pulsations wa: predicted to be relatively strong on the basis of the data of $\mathrm{MN}$, and because both the binary and 35-day phases (the latter just prior to X-ray turn-on) closely resembled those during which the reported detections of pulsed emission lines were made by DMM. The observations were made with the Lick Observatory $3 \mathrm{~m}$ telescope and image tube scanner (ITS) (Robinson and Wampler, 1972). As described in Chapter I, a 255-point digital spectrum was recorded every 10 ms on magnetic tape, with each point spanning a wavelength interval of $\sim 10 \AA$. The spectra covered the region $3500-6000 \AA$ and were calibrated and normalized with $\mathrm{He}-\mathrm{Ne}-\mathrm{Ar}-\mathrm{Hg}$ and quartz lamps and a standard star. In addition to the time-resolved data, higher resolution spectra (20 minute integrations) using all 2048 scanner channels ( 1 1 in width) were independently recorded * With the 4 " 
slit used the ITS had a resolution (FWHM) of $8 \AA$.

The higher resolution spectrum for the entire run is shown in Fig. III-1. Since the sky was not photometric for the entire run and the transparency was not monitored continuously, the normalization, particularly below $4000 \AA$, may not be precise. For reference, the shapes of representative blackbody curves are also shown. $\mathrm{HZ}$ Her is not isothermal at this binary phase, so detailed comparisons are not meaningful, but it may be useful to note that the spectrum can be characterized as roughly that of a blackbody with $\mathrm{T} \sim 14000^{\circ} \mathrm{K}$. The emission features $\lambda 4640$ and . $\lambda 4686$ are apparent, with equivalent widths of $\sim 2.0 \AA$ and $\sim 1.4 \AA$ respectively, and these lines are essentially constant in strength throughout the rum. (It is possible that the feature N III $\lambda \lambda 4634-4641$ is blended with C III $\lambda 4650$ [see Crampton and Hutchings, 1974]; we do not have sufficient resolution to distinguish these lines.) The prominent absorption features $H B$ and $H \gamma$ have equivalent widths of $\sim 6.8 \AA$ and $\sim 5.6 \AA$ respectively, and also persist throughout the run.

The amplitude, phase, and frequency of the broadband pulsations were determined from the time-resolved data as described in Chapter II. We found that the ratio of signal power to local power (the latter defined as the power averaged over an ensemble of independent frequencies in a region surrounding, but excluding, the frequency of interest) was 63.5 , corresponding to an $11(=\sqrt{2 \times 63.5})$ standard deviation effect. A portion of the power spectrum for $\mathrm{HZ} \mathrm{Her}$ is shown in Fig. III-2; the spike due to the $1.2 \mathrm{~s}$ pulsations is clearly visible (though not completely resolved in this figure). The (linear) change in the frequency with time was determined to be $\operatorname{small}\left(<10^{-8} \mathrm{~Hz} \mathrm{~s}^{-1}\right)$ and will not be considered 
further. The results of the broadband analysis are summarized in Table III-1 and are consistent with the data and mode1 described by MN.

The complex Fourier coefficients, $A_{k}(\omega), k=1,2, \ldots, 255$ were calculated for the 255 individual wavelength channels from Eq. (II.8) using the (angular) pulsation frequency determined from the broadband analysis. The individual signal phases were also determined from these coefficients according to Eq. (II.8). In the following, the individual phases have been measured relative to the broadband phase (arbitrarily chosen to be $0^{\circ}$ ); the broadband Fourier coefficient is thus real and positive.

The 255 complex coefficients were normalized according to a procedure similar to the one described in Chapter II, so that the uncertainties in both the real and imaginary parts of the normalized coefficients $A_{k}^{\prime}(\omega)$ are given by $\pm 1 / \sqrt{2}$. The situation here, however, turns out to be somewhat simpler than in the general discussion of Chapter II. As will be seen below, type II noise - noise that is correlated from wavelength channel to wavelength channel - is negligible in the $\mathrm{HZ}$ Her data. This is presumably due to the fact that sources of noise which are strongly correlated from channel to channel - fluctuations in seeing, transparency, and telescope guiding - have power spectra that fall roughly as $\mathrm{f}^{-1}$ and are thus unimportant at the relatively high frequency of $\mathrm{HZ}$ Her. We could not; however, have predicted this situation in advance.

We can verify that type II noise is indeed negligible by examining scatter plots of the $A_{k}^{\prime}(\omega)$ at frequencies near but excluding the frequency of interest. An example of such an off-frequency distribution of coefficients is shown in Fig. III-3a. In Chapter II it was noted that type I 
TABLE III-1. Broadband pulsations of $\mathrm{HZ}$ Her.

Start of run

End of run

Initia1 binary phase

Final binary phase

Power/local power*

Frequency*

Apparent

At solar system barycenter

Doppler velocity (relative to

$\mathrm{HZ} \mathrm{Her} / \mathrm{Her} \mathrm{X}-1$ center of mass) *

Average pulsed fraction ${ }^{\dagger}$
JD 2442686.615

JD 2442686.743

0.180

0.255

63.5
$0.807895(5) \mathrm{Hz}$

$0.807932(5) \mathrm{Hz}$

$-22(2) \mathrm{km} \mathrm{s}^{-1}$

$2.0(2) \times 10^{-3}$

Notes: Uncertainties $( \pm 1 \sigma)$ in least significant digit(s) indicated in parentheses.

Quantities with an asterisk $(*)$ have been calculated using only the first $2^{20} 10 \mathrm{~ms}$ data bins ( $-95 \%$ of data) as a calculational convenience.

${ }^{\dagger}$ Pulsed fraction is defined here with respect to the instantaneous light level, not the maximum light from the star as used in MN. The pulsed fraction would be $30-40 \%$ lower according to the latter convention. 
noise causes scatter in the $A_{k}^{\prime}(\omega)$ about the centroid of the distribution, while type II noise causes a displacement of the centroid from the origin. Figure III-3a is typical of the data near the frequency of interest in that the displacement of the centroid is very sma11; it is in fact consistent with what one would expect on the basis of the scatter of the points (type I noise) alone. It follows that the effects of type II noise can be neglected, a fact which we will find useful in the following paragraph.

To illustrate the connection between the distribution of the $A_{k}^{\prime}(\omega)$ at the pulsation frequency of $\mathrm{HZ}$ Her and the wavolength distribution of the pulsations, let us consider here two possibje extreme cases for the latter distribution. We sha11 assume, for simplicity, that each wavelength channel contains an equal number of photons. If the entire broadband signal, $\left|A^{\prime}\right|^{2}=63.5$, were to lie in a single wavelength channe $1, k_{0}$, then we would have $A_{k_{0}}^{\prime} \simeq \sqrt{63.5 \times 255} \simeq 127$ and the corresponding point in the scatter plot would lie along the real axis according to our above phase convention, with the remaining $A_{k}^{\prime}$ clustered about the origin. (The scaling law used here follows from the fact that the squares of the normalizing constants for the $A_{k}^{\prime}$ scale, in general, as the variances of the input distributions and therefore, in the absence of type II noise, roughly as the number of channels considered.) If, on the other hand, the signal were equally distributed among all wavelengths, then the entire distribution of the $A_{k}^{i}$ would be clustered about the point $\sqrt{63.5 / 25 \mathrm{~s}} \simeq 0.5$ on the real axis. That is, the centroid of the distribution would be displaced from the origin to this point. 
Results

The distribution of the complex Fourier coefficients calculated at the pulsation frequency of $\mathrm{HZ}$ Her is shown in Fig. III-3b. The most evident characteristic is a 0.6 unit displacement of the centroid of the distribution, suggestive of simple broadband pulsations of constant (wavelength independent) pulsed fraction; no excess of pulsations at any wavelength is apparent. To take account of the fact that the number of detected photons per wavelength interval varied substantially over the observed spectrum, we have subtracted the contribution of a constant pulsed fraction of $0.2 \%$ from each coefficient and the resulting distribution is shown in Fig. III-3c. The close similarity between Figs. III-3c and III-3a shows that, at least qualitatively, a fixed pulsed fraction, independent of wavelength, is a good description of the spectral distribution of the signa1.

Because of their special interest, the coefficients corresponding to the emission features $\lambda 4640$ and $\lambda 4686$ are shown separately (uncorrected for broadband contribution) in Fig. III-3d. It is clear that these coefficients do not have large components in the direction specified by the broadband pulse phase (positive real axis) and therefore the corresponding features do not contribute significantly to the observed pulsations. We have derived upper limits ( $90 \%$ confidence) of 0.08 and 0.09 for the pulsed fractions at any phase for the features $\lambda 4640$ and $\lambda 4686$ respectively. This corresponds to a total pulsed equivalent width of $\sim 0.3 \AA$, an order of magnitude smaller than that reported by DMM.

The foregoing analysis can be made more quantitative as follows. The 255 points on a graph of pulsed fraction versus wavelength were fitted 
successfully with no assumed wavelength dependence $\left(X^{2}=254.4,254\right.$ degrees of freedom), yielding a mean pulsed fraction $\overline{\hat{r}}$ of $2.0 \times 10^{-3}$. If 50 or more wavelength channels had no signal present, an unacceptably large $\chi^{2}$ would have resulted; the signal must therefore have been present in at least 200 of the wavelength channe1s. We also examined the hypothesis that the pulsed fraction varies linearly with wavelength, from $\bar{f}(1-\delta)$ at $4000 \AA$ to $\bar{f}(1+\delta)$ at $6000 \AA$. For this purpose we restricted the wavelength channels considered to those longward of $4000 \AA$ (because of the normalization problem noted above and the abundance of absorption features below this wavelength) and we also excluded those channels corresponding to the prominent absorption features $H \beta, H Y$, and $H \delta$; the remaining features in the spectrum represent on!y a small perturbation of the continuum light levels. We found an acceptable fit to this hypothesis $\left(X^{2}=184.7,187\right.$ degrees of freedom), with $\delta=0.18 \pm 0.15$, i.e., marginal evidence that the fractional modulation is stronger in the red than in the blue. Note that $\delta=0.2$ corresponds to the pulsed fraction increasing in direct proportion to the wavelength. It is not clear what value one expects for $\delta$ on theoretical grounds. The wavelength-dependence for the pulsed fraction varies from model to model among the several numerical models considered by Alme and wilson (1974) (Table 3 of their paper), although it is in general weak $(|\delta| \leqslant 0.1$ ). Anderson's (1977) numerical model is not carried to the point of a quantitative prediction for the pulsed fraction as a function of wavelength, but it is nonetheless clear that his results imply a positive value for $\delta$. In both cases the predictions appear to be consistent with the observations.

In addition to the above, we considered two simple models to explain 
the pulsed amplitudes as a function of wavelength in the continum. Suppose that the pulsations are due to a small sinusoidal temperature modulasion (of amplitude $\Delta T$ ) of a blackbody or thermal Bremmstrahlung source of light (characterized by a temperature T) whose time-averaged contribution is not necessarily the major component of light in the DC spectrum. If we denote the signal amplitude at wavelength $\lambda$ in photons (per unit time) per unit wavelength interval by $a(\lambda)$, then we have

$$
a(\lambda) \propto \frac{\partial}{\partial T}\left[\frac{1}{\lambda} F_{\nu}(\lambda, T)\right] \Delta T
$$

where $F_{v}$ is the usual source intensity per unit frequency interval. The DC spectrum, which may include substantial contributions of light which have nothing to do with the pulsations, is ignored in this model. We fitted the measured amplitudes (again excluding wavelengths below $4000 \AA$ and $H \beta$, $H \gamma$, and $H \delta$ ), corrected for detector response, to the above hypothesis, using the well-known expressions for the intensities of both blackbody and thermal Bremmstrahlung (free-free) sources. In the former case we found $\mathrm{T}_{\mathrm{bb}}=7000_{-1300}^{+2100}{ }^{\circ} \mathrm{K}$; in the latter, $\mathrm{T}_{\mathrm{ff}}=17000{ }_{-5000}^{+7000}{ }^{\circ} \mathrm{K}$, with an acceptable fit in each case. The blackbody hypothesis results in a value of the temperature winich is somewhat Iow compared to the color temperature of the DC spectiun. However, this does not necessarily mean that the incident $X$-ray energy is not thermalized in the atmosphere of HZ Her - the observed pulsations may represent an average over several thermal sources of different temperatures. In fact, if the X-rays are not thermalized (i.e. if the X-rays deposit their energy at small optical depths for the reprocessed radiation), then the weakness of the emission features (DC or puIsed) in HZ Her may prove difficult to understand (c.f. Tarter and McKee, 1973). 


\section{Discussion}

Because of the strong disagreement between our results and those of DMM, some further discussion is warranted. We first note that we have attempted to reproduce these latter results using equipment and analysis programs identical to those of DMM, but without success. Out of 10 nights of observations in 1975, a11 at appropriate binary phases, no statistical1y significant signals were seen through the narrow band interference filter covering $4620-4720 \AA$, although broadband pulsations were detected during four of the runs.

Our results might seem to indicate that the reported pulsed emission lines of DMM were transient events, which, for example, could result from the occasional presence of strongly pulsed soft $X$-rays. However, this interpretation leaves unresolved the reported phase agreement between the broadband and enission line pulsations of DMM. This phase agreement (within the $\pm 20^{\circ}$ experimental errors) is difficult to understand if the broadband pulsations are in fact distributed throughout the continuum (as our data indicates) and not simply due to other pulsing emission features (for example, filled-in Balmer absorption lines, as has been suggested). This can be seen as follows: One can show on general grounds (Avni: and Bahca11, 1974) that if the atmosphere of HZ Her requires a finite characteristic time $\tau$ to cool after $X$-ray heating, then the reprocessing efficiency (the ratio of the percentage optical modulation to the percentage $X$-ray modulation) will be given by

$$
n=\left(1+\omega^{2} \tau^{2}\right)^{-1 / 2}
$$

and there will be a corresponding phase shift (of the optical pulses 
relative to the $\mathrm{X}$-ray pulses) of

$$
\Delta \phi=-\tan ^{-1}(\omega \tau)
$$

where $\omega$ is the angular frequency of the modulation, $5.08 \mathrm{rad} \mathrm{s}^{-1}$. Equations (III.2) and (III.3) are true provided only that the atmosphere responds linearly to the incoming radiation. Now the low level of continuum pulsations from $\mathrm{HZ}$ Her can be understood if $n \approx 0.04$, allowing for a geometrical factor of $\sim 5$ which arises from differing light trave1 times over the surface of the star (see MN). This implies a cooling time of $\sim 5 \mathrm{~s}$, and a consequent phase $\operatorname{lag}$ of $88^{\circ}$ with respect to the incoming modulation, and is consistent with several theoretical values for $\tau$ in the range $1-10 \mathrm{~s}$ (Basko and Sunyaev, 1973; Dahab, 1974; and Alme and Wilson, 1974). On the other hand, the reported modulation in the emission lines of DMM is $\geq 25 \%$, which, again, allowing for the geometrical factor of 5 , implies $n \approx 1$ and hence $\tau \leq 0.1 \mathrm{~s}$, with a resultant phase lag of $\leq 30^{\circ}$. In other words, instead of phase agreement, one would expect pulsed emission 1 ines of the strength reported by IMM to have a phase $60^{\circ}$ to $90^{\circ}$ ahead of the phase of any continuum pulsations.

It should also be noted that the observations of DMM appear to disagree with spectroscopic data of other observers. Although the Doppler velocity and acceleration of the broadband signal of DMM establish that the observed pulsations come from the surface of $\mathrm{HZ}$ Her, Crampton and Hutchings (1974) present spectroscopic evidence which they feel suggests that both the He II and N III emission lines originate near the compact object (Her X-1) rather than HZ Her. In addition, they detect N III emission very near eclipse, which is difficult to explain if this feature originates from the heated surface of HZ Her. McClintock, Canizares, 
and Tarter (1975) have noted that one might expect He II emission from near the compact object by analogy with other, more easily observed, optica 1 counterparts of binary $X$-ray sources. We note that if these features came from near Her $X-1$ and were strongly pulsed, one would expect significant power at the appropriate Doppler shifted frequency; however in our data, the emission and absorpticil lines are not significantly pulsed at any nearby frequency. ${ }^{2}$. Additional data would be useful in clarifying the origin of the emission from this system.

The low pulsed fraction which we detect in the N III and He II emission lines raises another interesting problem. If a strongly modulated $X$-ray beam produces the emission lines in the upper atmosphere of $\mathrm{HZ} H e r$, then the recombination times are sufficiently low ${ }^{3}$ that the lines should appear $\sim 20 \%$ pulsed in contrast to our upper limit of $9 \%$. This disagrcement might be resolved in at least two ways. First, as noted above, the emission lines may originate near Her X-1 (e.g., in an accretion disk) instead of $\mathrm{HZ}$ Her. In this case, the expected pulsed fraction would depend upon the geometry. of the disk and could be quite low at this binary phase. Second, the $\mathrm{X}$-rays producing the emission 1 ines may not be strongly pulsed. Shulman et al (1975) have reported a substantia1 flux of soft $X$-rays with a low $(5-20 \%)$ pulsed fraction, and these soft $X$-rays should be most important in producing emission lines. However, these observations

As noted in the introduction, the broadband pulsations from the vicinity of Her $X-1$ are not typically seen at the binary phase under consideration here, but this does not preclude the possibility of pulsed lines from near Her $\mathrm{X}-1$ (since the equivalent width of the emission features is smal1):

3 According to the recombination coefficients and densities given in DMM, $\tau_{\text {He II }} \sim 10^{-2} \mathrm{~s}$ and $\tau_{\mathrm{N} \text { III }} \sim 10^{-3} \mathrm{~s}$ at optical depth unity. 
introduce problems of their own: as noted by the authors, this soft $X$-ray flux should excite emission lines whose strengths greatly exceed those actua1ly observed.

In summary, we have shown that the optical pulsations of $\mathrm{HZ}$ Her are distributed throughout the optical continuum. Although the details of this distribution are not precisely determined, our observations appear to be at least qualitatively consistent with the numerical results of other investigators. We find no evidence for pulsed emission lines and, on the basis of this experiment and the simultaneous spectra and pulsation data of JD 2441823 (discussed earlier), we can rule out the suggestion of DMN that a few prominent emission features are responsible for the pulsations. The reported pulsed emission lines of DMM may have been transient events, but their Doppler velocity and phase relative to the broailband pulsations do not fit well with other observations. We feel that any further investigations into the question of pulsed emission lines should address these latter issues. 
CHAPTER IV. TIME-RESOLVED SPECTROPHOTOMETRY OF DQ HERCULIS

\section{Intraduction}

The nova DQ Herculis (1934) has been an object of considerable renewed interest in the last five years. In addition to being a prototype for the classical novae, this star system is analogous in structure to the more recently discovered X-ray binaries, especially Her X-1 (HZ Her), but with a white dwarf replacing the neutron star of the latter (Lamb, 1974; Bath, Evans, and Pringle, 1974). Also the remarkable behavior of the optical pulsations from DQ Her near eclipse, the so-called $360^{\circ}$ phase shift discovered by Warner et al. (1972) (hereafter WPHN), undoubtedly provides an important clue to understanding this system, although the interpretation of this phenomenon (and, in fact, the interpretation of the optical pulsations themselves) is still a matter of controversy.

The time-resolved spectrophotometric observations of the present work (an investigation of the behavior of the pulsations as a function of wavelength) represents an attempt to gain insight into the puIsation mechanism in DQ Her. Katz (1975) has pointed out that such observations might elucidate the structure of DQ Her.

In the following, after summarizing the background material on this object, we shall present the results of our observations - most notably the detection of pulsations in He II $\lambda 4686$ and an unexpected phase shift (as a function of wavelength) across this line. We shall discuss briefly some general implications of the simple fact of the emission line pulsations and sha11 then present a rather idealized geometrical model which makes clear the connection between the emission line phase shift 
and the eclipse-associated phase shift of WPHN. This mode1 is based on the idea that the pulsations, excited by hot spots on a rotating white dwarf, arise either i) at the inner edge of the azcretion disk as suggested by Katz (1975) and by Rees (1974), or ii) from the surface of the disk - predominantly the back half of the surface - as suggested by Herbst, Hesser, and Ostriker (1974). We shall also consider the implications of our results for a number of other models proposed for DQ Her and noted below. Finally, an important distinction between our interpretation of the eclipse-associated phase shift and Katz's (1975) interpretation of the same phenomenon will be discussed.

\section{Background}

We shall begin with a summary of the relevant background material on DQ Her and a brief description of the various models which have been proposed for this system.

DQ Her was observed to be an eclipsing binary with a periLod of $4^{h} \dot{3}^{m}$ by Walker (1954). Greenstein and Kraft (1959) found velocity variations in He II $\lambda 4686$ and members of the Balmer series indicative of orbital motion with $K \sim 150 \mathrm{~km} \mathrm{~s}^{-1}$. These authors also found that the above He II line shows a classical rotational disturbance before and after mid-eclipse; that is, the central wavelength of the emission line is displaced sharply longward during ingress and sharply shortward during egress. This effect provides direct and convincing evidence for an accretion disk in $D Q$ Her and establishes that the $\lambda 4686$ emission originates in the disk (an important fact for this work). The current view is that the system consists of a hot white dwarf surrounded by an accretion disk (the latter being responsible for most of the light from the system) and 
a dark companion star which overflows its Roche lobe (Kraft, 1959). Optical pulsations from this system with an amplitude of a few percent and a very stable period of about $71 \mathrm{~s}$ were discovered by Walker (1956). The pulsations disappear near mid-eclipse; the duration of the disappearance is not precisely known, but it is believed to be about five cycles (WPHN). Herbst, Hesser, and Ostriker (1974), combining all the pulsation data collected from 1954 to 1971 , have established that the pulsation period is highly stable, though steadily decreasing on a timescale, $|P / \dot{P}|$, of $2.64 \times 10^{6}$ years. In other words, the clocking mechanism in DQ Her drifts by only about 1 part in $10^{12}$.

In addition to the $71 \mathrm{~s}$ periodicity in the intensity of DQ Her, some (rather marginal) evidence for a modulation of twice the period (142 s) has been reported in both Iinear and circular polarization (Kemp et al., 1974 ; Swedlund et al., 1974).

As mentioned in the Introduction, WPHN have discovered a surprising aspect to the behavior of the pulsations near eclipse: Out of eclipse the pulsation phase is roughly constant in time (though see Patterson et al. [1977]), as one would expect from a signal of constant frequency, but near the onset of eclipse the phase begins to advance and continues to do so until the pulsations disappear, by which point it has gained a substantial fraction of a cycle. Near the end of eclipse, when the pulsations reappear, the phase appears retarded by a similar degree and then advances until it reaches the same constant value it had prior to eclipse. For historical reasons this effect is often referred to as a (positive) $360^{\circ}$ phase shift; however, we shall instead adopt the terminology of Nather and Robinson (1974) and refer to it below as the "eclipse-associated phase shift." 
Both Walker (1958) and Kraft (1959) have attributed the 71 s pulsations to radical oscillations of the degenerate star. However, it is difficult to explain the eclipse-associated phase shift in terms of this mechanism and, as a result, several other models have been put forth. WPHN have considered a model involving a grazing eclipse of a white dwarf oscillating in an $\ell=2$ mode, but the model is artificial and not entirely successfu1. The mode1 proposed by Nather and Robinson (1974) for both UX Ursa Majoris and DQ Her involves a white dwarf oscillating in a nonradial g-mode with $\ell=2$ and $m= \pm 2$. In this case the pulsed radiation is not observed directly but is reprocessed in a greatly extended optically thick photosphere which is suspended by Keplerian motion. The details of how the white dwarf oscillations are coupled to the light curve, however, remain poorly understood in this mode1.

Bath, Evans, and Pringle (1974) examine in some detail an oblique magnetic rotator model consisting of a white dwarf rotating with a $142 \mathrm{~s}$ period and possessing two hot spots due to accretion at the two magnetic poles. The problem with this model (as its authors acknowledge and as we shall explain below) is that the pulsation phase should decrease during ingress, appear advanced as the eclipse ends, and then decre $\Rightarrow$ again until it reaches its normal value (a phase shift of $-360^{\circ}$ in the terminology of WPHN), exactly opposite to what is observed. Rees (1974) points out, in a note added in proof to the above paper, that the correct sign for this effect could be obtained if the pulsations were due to a spot (or two spots) on the inner edge of the accretion disk, rather than on the white dwarf itself; although such an inner edge effect predicts a phase shift more rapid than that actually observed. 
Lamb (1974) discusses an oblique rotator model similar to that of Bath et al. (1974), but in Lamb!s picture the eclipse-associated phase shift is due essentially to an artifact of the data-analysis procedures: Following a suggestion by WPHN, Lamb argues that the phase shifts during ingress and egress are a consequence of fitting a sine wave $f$ decreasing and increasing amplitude respectively with a constant amplitude sine wave. However, we show in the Appendix of the current work that this cannot be the case: Any (artificial) phase shift induced by the fitting procedures of WPHN must have been sma1l, almost certainly less than $5^{\circ}$, and in addition, the sign of such a shift is not necessarily positive (as required by Lamb).

Herbst, Hesser, and Ostriker (1974) note that the $\gtrsim 500 \mathrm{~s}$ duration of ingress and egress of the eclipse of the pulsating region (or, alternatively, the duration of the phase advance during ingress and egress) from the data of WPHN and the $150 \mathrm{~km} \mathrm{~s}^{-1}$ radial velocities of Greenstein and Kraft (1959) imply that the dimensions of the pulsating region are $\sim 10^{10}$ $\mathrm{cm}$, an order of magnitude too large for a white dwarf. These authors infer that the pulsations must therefore originate in the accretion disk, and go on to suggest, independently, a model similar to that of Rees, in which radiation from the white dwarf hot spots periodically excites the back side of the disk (a region conceivably extending well beyond the inner edge).

Katz (1975) also discusses the oblique rotator model, but suggests a different mechanism for the eclipse-associated phase shift. In Katz's picture, the pulsations arise from a reflection effect in which the uItraviolet radiation from the rotating white dwarf is absorbed by and reradiated from two distinct regions: i) matter fixed in configuration 
with respect to an inertial observer, e.g., the inner edge of the accretion disk, and ii) matter fixed in the co-rotating frame, e.g., the companion star. This gives rise to pulsations at two different frequencies (analogous to solar and sidereal days), whose interference leads to the observed phase shift at eclipse.

We sha1I discuss our results below in terms of a model based on the three mode1s - developed by Rees (1974), Katz (1975), and Herbst, Hesser, and Ostriker (1974) - which involve an oblique magnetic rotator and reprocessing in the accretion disk.

Details of the Observations and Data Reduction

We recorded spectra of DQ Her for a 4.5 hour period on 1976 June 23, beginning at ID 2442952.755 and covering very nearly one complete binary cycle (binary phase interval $0.56-1.54$ ). The observations were made with the Lick Observatory $3 \mathrm{~m}$ telescope and image-tube scanner (ITS) (Robinson and Wampler, 1972). As described in Chapter I, a 255-point digital spectrum was recorded every $10 \mathrm{~ms}$ on magnetic tape, with each point or channel spanning a wavelength interval of about $10 \AA$. The total wavelength band covered was $3600-6100 \AA$. These $10 \mathrm{~ms}$ spectra were later summed 200 at a time so that the resulting data consisted of a string of $2^{13}(=8192)$ contiguous 2 s spectra of 255 wavelength points each. In addition to the time-resolved data, 13 higher resolution spectra (20 minute integrations) using a11 2048 scanner channels ( $\sim$ 1 in width) were independently and simultaneously recorded. We measured the width (FWHM) of the prominent emission line features in the night sky to be $16 \AA$, corresponding to a resolution $(\alpha)$ of $7 \AA$. However, the resolution of these sky features 
is determined by the full width (7") of the spectrograph aperture; the resolution for stellar features is determined (for large apertures) by the size of the seeing disk. We estimated this latter resolution ( $\sigma$ ) to be $4 \AA$, based on the observed disk size and a previous aperture calibration. In addition, the orbital motion of DQ Her during our observations caused (unremoved) wavelength shifts of up to $\pm 2 \AA$, thus increasing our effective resolution to $\sigma \simeq 5 \AA$.

In addition to (and immediately preceding) the optical observations under discussion here, we made a brief run on the object with the same equipment, but at longer wavelengths, extending into the infrared:

$6100-8600 \AA$. The binary phase interval covered in this shorter run was $0.43-0.55$. Unless otherwise stated, the results below apply only to the longer run, covering the wavelength band $3600-6100 \AA$.

In order to investigate the broadband $71 \mathrm{~s}$ signal from DQ Her, the number of photons in each $2 \mathrm{~s}$ spectrum were sumed over wavelength to give the broadband count rate as a function of time. The resulting function was smoothed by fitting each 20 minute segment to a cubic polynomial and then dividing out the polynomial. In order to eliminate the grosses nonstatistical fluctuations in the count rate (the majority of such excursions presumably resulting from telescope guiding variations), the $4 \%$ of the points which were furthest from their expected values on the basis of the above fit were deleted and replaced with their expected values. This procedure substantially improved the signal-to-noise ratio. To avoid the complicating effects of the eclipse-associated phase shift and the rapid intensity variation during eclipse, all points during the $\sim 40$ minute eclipse (for our purposes from binary phase 0.92 to 1.08 ) were also 
deleted. The broad band smoothing function described above was also used to smooth the 255 individual wavelength sets. The 2 s spectra corresponding to deleted broad band data points were likewise deleted and replaced by the average $2 \mathrm{~s}$ spectrum. The puised fractions quoted below have, of course, been corrected for such deletions.

The 2048-point spectrum for the binary cycle covered in our observations (excluding the eclipse) is shown in Fig. IV-1. The lines of the Balmer series and of he II $\lambda 4686$ are clearly visible in emission. Weaker emission features of N III - C III at about $\lambda 4640$, C II $\lambda 4267$, and He I $\lambda 5875$ are also present. The forbidden feature of [0 II] at $\lambda 3727$ is evidence of the well-known nebula surrounding DQ Her (c.f. Greenstein and Kraft, 1959). This nebula is largely irrelevant for the present work; we are chiefly concerned here with the accretion disk. However, the possible presence of a nebular component to emission lines which also originate in the disk may represent a complication to the use of these lines as a probe of conditions in the disk.

The continuum light from DQ Her is characterized by a notably flat spectrum as is evident from Fig. IV-1. This flatness manifests itself as peculiar U-B and B-V colors (c.f. Walker, 1956). Kraft (1959) has suggested that this continuum shape can be explained as due to Bremsstrahlung emission from an optically thin disk. However, a more recent view is that the accretion disks of cataclysmic variables are, in general, optically thick (Robinson, 1976b). The accretion disk model of Pringle and Rees (1972), although discussed in the context of the $X$-ray binaries, is applicable here. In this case the spectral flatness results from a superposition of a large number of blackbody curves of different 
temperatures corresponding to different radii in the disk; one finds that the spectrum is given approximately by $F_{v} \propto v^{1 / 3}$. Edge effects may change the details of the continuum spectrum, which is also somewhat sensitive to the mass accretion rate. Such effects may explain why the continuum in Fig. IV-1 slopes slightly toward the red, while a true $v^{1 / 3}$ spectrum would slope slightly toward the blue.

Our eclipse data (not shown) exhibit the rotational disturbance of the He II line discussed by Greenstein and Kraft (1959), and show the effect in the Balmer series as well. However, due to the long integration times ( 20 minutes) in our higher resolution spectra, it is difficult to analyze this effect in a quantitative way. The data of Greenstein and Kraft also suffer from insufficient time resolution. These authors conclude on the basis of this effect that the (effective) disk velocity exceeds $350 \mathrm{~km} \mathrm{~s}^{-1}$, though by how much is not clear. We shall return to the question of disk velocities from a different point of view later on. The broadband data were Fourier transformed using a standard FFT algorithm. A portion of the resulting power spectrum is shown in Fig. IV-2; the spike due to the $71 \mathrm{~s}$ pulsations is clearly visible (though not completely resolved in this figure). Lower frequency spikes may be due to fluctuations in seeing and transparency, and to telescope guiding; it is not clear that any given lower frequency spike is associated with the star itself (although the system is known to exhibit low frequency flickering). The evident lack of power at the lowest frequencies is a result of the smoothing procedure described above.

The precise amplitude, phase and frequency of the broadband pulsations were determined from the time-resolved data as described in Chapter II. We found that the ratio of signal power to local power (the latter defined 
as the power averaged over an ensemble of 100 independent frequencies in a region surrounding, but excluding, the frequency of interest) was 27.4 , corresponding to a $7(=\sqrt{2 \times 27.4})$ standard deviation effect. It is interesting to note that if our uncertainties resulted solely from counting statistics, the observed modulation would have corresponded to about a 40 standard deviation effect; clearly nonstatistical uncertainties dominate the broadband data at this 10 frequency.

Our measured value for the pulsation frequency is $14.073(5) \mathrm{mHz}$, consistent with the value $14.072 \mathrm{mHz}$, extrapolated to the epoch of our observations from Eq. (6) (1inear terms riliy) of Herbst, Hesser, and Ostriker (1974). ${ }^{4}$ The broadband phase used implicitly in the following analysis was determined with respect to the latter, more accurate, frequency. Our value for the pulsed fraction is $0.9 \% \pm 0.1 \%$, consistent with the average value seen by WPHN (as determined from Rigs. 2 and 5 of their paper), though a factor of 2 lower than Walker's $(1958,1961)$ earlier observations. We note that long time-constant decays in the ITS phosphor might tend to reduce the amplitude of the signal; however we have shown that we can easily resolve the $1.24 \mathrm{~s}$ optical pulsations of $\mathrm{HZ}$ Her (a star of brightness comparable to that of DQ Her) with the same equipment (c.f. Chapter III), so any such smearing-out effects should be unimportant on the substantially longer timescales dealt with in the present work.

The complex Fourier coefficients, $A_{k}(\omega), k=1,2, \ldots, 255$, defined as in Chapter Ir, were calculated for the 255 individual wavelength channe1s from Eq. (II.12), with the frequency of the signal again taken to be

${ }^{4}$ The barycentric correction is negligible at this low frequency. 
14.072: $\mathrm{mHz}$. The individual signal phases were similarly determined from these coefficients according to Eq. (II.12). In the following the individual phases have been measured, as usual, relative to the broadband phase (arbitrarily chosen to be $0^{\circ}$ ); the broadband Fourier coefficient is thus real and positive.

The 255 Fourier coefficients were then normalized according to the procedure described in Chapter II. The ensemble of frequencies used for this purpose was the same as that used to determine the uncertainties in the broadband analysis. For the observations of DQ Her, unlike those of $H Z$ Her, type II noise (noise that is strongly correlated from wavelength channel to wavelength channel) was not found to be negligible compared to the more familiar uncorrelated noise (type I). Since the differential refraction in the atmosphere was small for these observations (an average of $0 . " 6$ between the extreme ends of the observed spectrum), the star image was subject to the same sources of type II noise in different wavelength bands, and the assumption that type II noise was $100 \%$ correlated from channel to channel is a reasonable one. We assumed in addition that type II noise was directly proportional to the number of photons counted in any given channel (see Chapter II), though our final results are not very sensitive to the exact behavior. We made no assumptions regarding the count-rate dependence of type I noise.

The various distributions of normalized coefficients $A_{k}^{\prime}(\omega)$ shown in Fig. IV-3 have thus been normalized such that the uncertainties due to type I noise alone in both the real and imaginary parts of any given point are $\pm 1 / \sqrt{2}$. The uncertainties associated with the phases and amplitudes derived from these cuafficients in the following have been determined 
from the above uncertainties by the propagation of errors. The errors due to type II noise have been folded in where appropriate. In general, in this data type I noise dominates in the individual wavelength bands, but, due to the stronger rate dependence of type II noise, the latter dominates in the broadband data. We could not, however, have predicted this situation in advance.

\section{Results}

The scatter plot of the 255 normalized Fourier coefficients at the frequency of DQ Her is shown in Fig. IV-3b. As indicated above, the orientation of the axes has been chosen according to our convention that the broadband coefficient lies along the positive real axis $\left(0^{\circ}\right.$ phase) It is clear that, to first order and with respect to a typical ensemble of off-frequency coefficients (Fig. IV-3a), the entire on-frequency distribution has been displaced substantially to the right (in the direction of the broadband signa1), and consequently the optical pulsations in DQ Her are distributed throughout the wavelength band $3600-6100 \AA$. In the following we shall examine the details of this distribution. We shall first consider continuum wavelength channels, containing little or no light from emission lines, and secand, channels involving substantial emission line contributions.

We determined the pulsation amplitude in each of the 211 continuum channels by considering only the real part of the appropriate Fourier coefficient. In this way only signals in phase with the net broadband signal were considered; such a restriction effectively filters out the component of the noise orthogonal to the signal. In order to check that 
no loss of information resulted from this procedure, we examined the scatter, $s_{\perp}^{2}$, in the imaginamy parts of the (normalized) coefficients, defined as:

$$
s_{\perp}^{2}=\sum_{k}\left(\operatorname{Im} A_{k}^{\prime}\right)^{2}
$$

where the sumnation index $k$ runs over the wavelength channels considered. The subscript added to $s_{\perp}^{2}$ indicates that this quantity is restricted to components orthogonal to the broadband signal. We found $s_{\perp}^{2}=101.8$, which falls within the range of values $105.5 \pm 10.3$ expected on the basis of statistical fluctuations (type I noise) alone. In other words, there is no evidence for any variation of the signal phase across the continuum. We should emphasize here that the quantity $s_{\perp}^{2}$ is useful in this statistical test of the data, but its numerical value is not a quantitative measure of any intrinsic property of the star.

In the following we shall je concerned both with signal amplitude and with pulsed fraction, $f$, the latter defined by $f=a / r$, where $r$ is che average photon coumt rate and $a$ is the signal amplitude in the same units. In dealing with the signal amplitude (a) we must correct for the wavelength dependent detector response: pulsed fraction (f), however, is not affected by this response.

We obtained a good fit to the continuum data with the simple hypothesis that the pulsed fraction was constant, independent of wavelength: The value of $\chi^{2}$ was 211 for the 210 degrees of freedom, and the mean pulsed fraction, $\bar{f}$, was $0.92 \% \pm 0.12 \%$. When we assumed instead that the pulsed fraction in the continuum varied linearly with wavelength, from $\bar{f}(1-\delta)$ at $3600 \&$ to $\bar{f}(1+\delta)$ at $6100 \AA$, then we found that the fractional modulation was 
marginally stronger in the blue than in the red: $\delta=-0.077 \pm 0.054$.

We also examined a simple model to explain the pulsation amplitudes as a function of wavelength in the continuum. Suppose that the pulsations are due to a small sinusoidal temperature variation (amplitude $\Delta T$ ) of a blackbody or thermal Bremsstrahlung source of light (characterized by temperature T), whose time-averaged contribution is not necessarily the major component of light in the DC spectrun. The amplitude of the pulsations at a given wavelength will then be proportional to the difference between i) the flux of photons of that wavelength from the source when the temperature is $T+\Delta T$, and $i i)$ the $f 1 u x$ when the temperature is $T$. We have:

$$
a(\lambda) \propto \frac{\partial}{\partial T}\left[\frac{1}{\lambda} F_{v}(\lambda, T)\right] \Delta T
$$

where $F_{\nu}$ is the usual source intensity per unit frequency interval. The DC spectrum, which may include large contributions of light which have nothing to do with the pulsations, is ignored in this model.

We fitted the measured signal anplitudes (corrected for the detector response) to the above hypothesis, using the well-known expressions for the intensities of both blackbody and thermal Bremsstrahlung (free-free) sources. In the former case we found $\mathrm{T}_{\mathrm{bb}}=7700 \pm 600^{\circ} \mathrm{K}$; in the 1atter - $T_{\text {ff }}=19000 \pm 2000^{\circ} \mathrm{K}$, with an acceptable fit in each case. For comparison, we note that Kraft (1959) has estimated (though with considerable uncertainty) that the temperatures of the white dwarf and disk are $8 \times 10^{40} \mathrm{~K}$ and $2 \times 10^{4} \mathrm{~K}$, respectively. Thus the "pulsation temperature" for the free-free fit is perhaps typical of the system, but if the pulsations are due to a single blackbody source, then this must be located in a relatively 
cold part of the system. Of course, more complicated models, e.g., a sup?rposition of blackbody sources, analogous to the case of the DC spectrum, are also possible.

Our near infrared observations, near binary phase 0.5 , establish that the pursations extend into the wavelength band $6100-8600 \AA$. We found a mean pulsed fraction $\bar{f}=0.80 \% \pm 0.19 \%$ in this region of the spectrum. Due to the shorter duration of this run, the uncertainty in the slope of the pulsed fraction versus wavelength is relatively large. When we assumed that the pulsed fraction in the continuum varied linearly from $\bar{f}(1-\delta)$ at $6100 \AA$ to $\bar{f}(1+\delta)$ at $8600 \AA$, we found $\delta=-0.52 \pm 0.28$. Because there is evidence that the pulsed fraction in DQ Her varies irregularly (Wa1ker, 1958,1961; Nather and Wamer, 1969), we have not combined this lata with the optical data described above into a single fit. The only prominent emission feature in this long wavelength band is $\mathrm{H} \alpha$; it does not show any significant enhancement of the pulsation amplitude above the underlying continuum.

The pulsation amplitudes in the 44 emission line channels were also determined from the real parts of the appropriate Fourier coefficients, analogous to the det.smination of the continuum amplitudes, except that these coefficients were first corrected for the underlying continuum by subtracting off the Fourier coefficient expected from the continuum alone on the basis of the two-parameter fit described above. The pulsed fractions obtained by this procedure for the ten most prominent emission lines are presented in Table IV-1. Errors in the pulsed fractions quoted include a contribution from an uncertainty of $2 \%$ in estimating the level of the underlying continuum. The errors are dominated by cúnting statistics 
TABLE IV-1. Pulsed fractions in emission features in DQ Her.

\begin{tabular}{|c|c|c|c|}
\hline Species & Wavelength & $\begin{array}{l}\text { Pulsed } \\
\text { fraction }(\%)\end{array}$ & $\begin{array}{c}\text { Error } \\
\left(\frac{\mathrm{o}}{6}\right)\end{array}$ \\
\hline$\left[\begin{array}{ll}0 & \mathrm{II}\end{array}\right]$ & 3727 & -1.4 & 2.8 \\
\hline $\mathrm{H} \zeta$ & 3889 & -0.9 & 3.4 \\
\hline $\mathrm{H} \epsilon$ & 3970 & -0.9 & 1.8 \\
\hline Hठ & 4101 & -0.4 & 0.9 \\
\hline C II & 4267 & +1.5 & 2.6 \\
\hline $\mathrm{H} \gamma$ & 4340 & -0.5 & 0.7 \\
\hline N III/C III & $\sim 4640$ & +2.5 & 0.9 \\
\hline $\mathrm{He}$ II & 4686 & +2.3 & 0.4 \\
\hline$H \beta$ & 4861 & -0.6 & 0.4 \\
\hline He I & 5875 & -3.4 & 2.0 \\
\hline
\end{tabular}

Notes: Pulsed fractions have been corrected for contributions due to underlying continuum; uncertainties include the effects of a $2_{\%}^{\circ}$ error in estimating the level of this background. Signals have been restricted to the components in phase with broadband pulsations; as a consequence negative pulsed fractions can result. 
and are thus larger in the weaker features and near the ends of the spectrum as a result of low detector response and/or extinction in the earth's atmosphere.

The results indicated in Table IV-1 are relatively simple: The line He II $\lambda 4686$ and the $N$ III - C III feature at $\lambda 4640$ are pulsed in considerable excess of the underlying continuum, while pulsations in the Balmer series are suppressed and, in fact, consistent with not being present at all. The contrast between $\lambda 4686$ and $H \beta$ is somewhat surprising, especially since both our sensitivity to and the intrinsic strength of these two features is comparable. This difference can be understood, however, in terms of the difference.in ionization potential between He II and $H I$. This point will be discussed in more detail below.

Because of the good statistics and strong signal in the $\lambda 4686$ line, it is appropriate to examine the Fourier coefficients of this feature in more detail. These coefficients, corrected for the underlying continuum, have been plotted in Fig. IV-3d. Again, as iil Figs. IV-3a and 3b, the axes have been rotated so that the broadband coefficient falls along the positive real axis. Note that for the single emission line, there are five coefficients (numbered in order of increasing wavelength), corresponding to the fact that in our data the wings of the line extend over about $50 \AA$, or 5 of the $10 \AA$ wavelength channels. This spread is due to the combined effects of intrinsic (presumably rotational) line width and our effective resolution of $\sigma \simeq 5 \AA$. Unlike the continuum case, the scatter in the imaginary part of these coefficients exceeds what one would expect on the basis of statistics. For this scatter, defined as in Eq. (IV.1), we find $s_{\perp}^{2}=11.1$, well above the range $2.5 \pm 1.6$ expected on the basis of statistics alone. 
In order to check that this excess scatter is a real effect, we repeated the above procedure, calculating the Fourier coefficients at a different frequency for the same emission line. For this purpose we chose a frequency of $8.348 \mathrm{mHz}$, corresponding to the Iarge spike evident to the left of the signal spike in the power spectrum of Fig. IV-2; this spike is presumably due to noise. The five corrected coefficients of this off-frequency analysis are shown in Fig. IV-3c; there is no evidence of excess scatter in the imaginary direction $\left(s_{1}^{2}=2.6\right)$. Note that the continuum corrections which we have made involve only the real parts of the Fourier coefficients and cannot contribute to the large value of the scatter observed in the imaginary parts.

The excess scatter in the $\lambda 4686$ Fourier coefficients orthogonal to the direction of the broadband signal is evidence for a phase variation across the emission line. This can be seen more directly in Fig. IV-4a, in which we have plotted the phase of the $71 \mathrm{~s}$ pulsations for each of the five $\lambda 4686$ channels, with phase $0^{\circ}$ defined by the phase of the continuum pulsations. These phases (and their errors) are obtainable directly from the (continum corrected) complex coefficients plotted in Fig. IV-3d. Figure IV-4a shows a striking and systematic effect: The pulsation phase in $\lambda 4686$ increases rapidly with increasing wavelength across the emission 1ine. Furthermore, the He II line center is in phase with the continuum pulsations. We can describe this effect equivalently by noting that in the short wavelength wing of the line the pulsations lag the continuum, while in the long wavelength wing the pulsations lead the continuum. The pulse amplitudes in the five channels are shown in Fig. IV-4b. Due to the phase variation across the line, these amplitudes have been 
calculated using both the real and imaginary parts of the appropriate Fourier coefficients (unlike Table IV-1 or the analysis of the continuum channels in which only the real parts were used). Because the interpretation of the phase variation across the emission line is much more transparent than the corresponding amplitude variation, we shall concentrate on the former in the discussion beiow. Before leaving Fig. IV-4b, however, we should point out that we would have expected (and, unfortunately, the simple geometrical models which we will consider below predict) the pulsation amplitudes to be symmetric about the central wavelength, while Fig. IV-4b indicates that the "center of pulsations" in the emission line is displaced toward longer wavelengths. It is curious that Kraft (1959) has suggested, on the basis of different evidence, that near eclipse the center of light of the accretion disk may be displaced similarly toward the receding or red-shifted half of the disk. It seems likely that these two displacements are related, although it is unclear why no asymmetry corresponding to that of Fig. IV-4b is evident in the (DC) line profile, which is centered, in our data, to within about $1 \AA$ of the true line center at $4686 \AA$. We shall leave this matter unresolved here, although we note that this lack of a corresponding asymmetry in the DC line profile may indicate that the pulsed and DC components of $\lambda 4686$ do not have identical origins.

It is important to state here that due to the rather $10 \mathrm{w}(\sigma \simeq 5 \AA)$ resolution of our spectra, one must be rather cautious about any quantitative interpretation of Figs. IV $-4 a$ and $4 b$. That is, for example, the fourth wavelength bin, extending from $4692 \AA$ to $4702 \AA$, also includes substantial contributions from photons whose true (as opposed to measured) wavelengths lie in the range corresponding to the third wavelength bin, $4682 \AA$ to $4692 \AA$. 
However, even allowing for this potential resolution problem, the extent of the emission line effect in $\lambda$ in the above figures suggests that velocities substantially larger than the orbital velocity of the system (150 $\mathrm{km} \mathrm{s}^{-1}=0.23$ wavelength channels) are involved. ${ }^{5}$ This indicates that the effect, like the rotational disturbance discussed earlier, originates in the accretion disk. A somewhat less obvious but equaliy important point is the following: It is difficult to explain the phase variation across the line by any disk model which does not invoke a strong front/back asymmetry in the disk. Perhaps the simplest way to build such an asymmetry into a mode 1 for $D Q$ Her is to have the pulsations originate at the inner edge of the disk; a second possibility is that, due to the combined effects of the disk shape and orbital inclination, the back half of the disk aurface is more visible than the front half. We shall return to these points below, after a preliminary discussion involving some physical implications of the existence of pulsations in He II as well as their absence in $H I$.

\section{Discussion}

\section{Physical Considerations}

The difference noted above between the hydrogen and helium pulsation strengths can be accounted for in terms of the discussion which follows. First, however, let us assume that the mechanism responsible for populating the upper levels of He II is electron captured onto He III (Kraft, 1959), and similarly for hydrogen. For consistency. we need to establish that

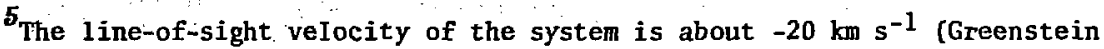
and Kraft, 1959) and consequently completely negligible here. 
the helium recombination time is short compared to $71 \mathrm{~s}$, otherwise the pulsations would be washed out and not observable. We take representative values for the electron temperature and density in the disk from Kraft (1959) to be $\mathrm{T}_{\mathrm{e}}=2 \times 10^{40} \mathrm{~K}$ and $\mathrm{n}_{\mathrm{e}}=3 \times 10^{13} \mathrm{~cm}^{-3}$, respectively. For this temperature the coefficient for recombination onto the $n=4$ level of He II (restricted to ions which subsequently decay to $\mathrm{n}=3$ ) is $\propto($ He II $\lambda 4686)=$ $1.2 \times 10^{-13} \mathrm{~cm}^{3} \mathrm{~s}^{-1}$ (Seaton, 1968). Thus we have for the recombination time $\mathrm{t}_{\mathrm{He}}=\left(\alpha_{\mathrm{n}}\right)^{-1}=0.3 \mathrm{~s}$, much smaller than $71 \mathrm{~s}$, as required. Similarly for $H \beta$ we have $\alpha(H \beta)=1.6 \times 10^{-14} \mathrm{~cm}^{3} \mathrm{~s}^{-1}$ (Seaton, 1968), and $t_{H}=2 \mathrm{~s}$, also $\ll 71 \mathrm{~s}$; the lack of pulsations in $H \beta$ cannot be due to temporal smearing-out effects. (Robinson [1976b] has suggested that the disk electron density may substantially exceed Kraft's [1959] estimate; however, the conclusion that the recombination times are much less than $71 \mathrm{~s}$ is not affected.) From the above recombination times for helium, Kepler's 1aw, and a white dwarf mass of $\sim 1.1 \mathrm{M}_{0}$ (Robinson, 1976a), it follows that an ion of He III in the accretion disk traverses a negligible fraction of its orbit around the disk from the time it is created uritil it recombines and produces a photon at $\lambda 4686$. We will therefore be justified in treating the ionization and recombination process (as we shall in the following section) as occurring at a point.

Now if the emission 1 ines are in fact recombination-driven and since the recombination times must be short, it follows that in a given volume element of the disk the observed periodicity in the $\lambda 4686$ intensity must be due to a corresponding modulation of the recombination rate, $r=n_{e} n_{\text {He III }} \alpha_{\text {He II }}{ }^{(T)}$. This in turn must be due to a modulation of one or more terms on the right-hand side of this equation. However, the 
electron density, $\mathrm{n}_{\mathrm{e}}$, appears in the hydrogen recombination rate as wel1, and in addition, the temperature dependence of the coefficients for recombination onto $H$ II and $H$ III is virtually the same $\left(\sim T^{-\frac{1}{2}}\right)$. Thus, barring very large temperature varjations, the most important modulation of the three terms must be that of $n_{H e}$ III, the number of alpha particles per unit volume; otherwise the effect would show up to a similar degree in the Balmer series as we11. The modulation of this factor simply reflects a corresponding modulation of the ratio $n_{\mathrm{He} \mathrm{III}} / n_{\mathrm{He} \mathrm{II}}$. The lack of an observed effect in hydrogen can then be understood if the hydrogen in the pulsating region is largely or completely ionized throughout the entire 71 s cycle. Recombination radiation will still be produced in the Balmer series, but if $n_{\mathrm{HII}} / \mathrm{n}_{\mathrm{HI}} \gg 1$ at all times, then the rate of hydrogen recombination will not vary appreciably over the pulse period. Due to the large difference in ionization potential between He II (54.4 eV) and H I $(13.6 \mathrm{eV})$, this difference in the modulation of the hydrogen and helium ion populations is not surprising. The idea that the emission line pulsations should therefore tend to show up in species with higher ionization potential is supported by the significant pulsation excess in N III C III (see Table IV-1, page 53); these species have ionization potentials of $47.4 \mathrm{eV}$ and $47.9 \mathrm{eV}$ respectively.

We note for the sake of completeness that the situation in the Balmer series may be somewhat complicated due to the possibility of selfabsorption of these lines in the disk (Kraft, 1959) and the presence of a nebular component to these 1ines. However, since the Balmer series do show some evidence of rotational disturbance as well as substantial diminution during eclipse, the disk component of these lines cannot be 
negligible and it is unlikely that the above difference in the pulsations of the hydrogen and helium emission lines reflects simply the difference between conditions in the nebula and disk.

The oblique magnetic rotator mode1 of DQ Her provides a natura1 explanation for the periodic ionization of He II: At an arbitrary point on the disk there is a flux of ultraviolet photons originating at the hot spots around the magnetic poles of the white dwarf, and this flux varies as the star rotates. At least the high-energy tail (and possibly more) of this photon distribution is capable of ionizing He II. Bath, Evans, and Pringle (1974) estimate the characteristic energy from these hot spots at between 20 and $250 \mathrm{eV}$. One could imagine that a similar situation arises in the case of nonradial oscillations (as in the mode1 of Nather and Robinson [1974]). If we suppose that these oscillations are accompanied by a temperature variation, then the number of ionizing photons in the tail of the (local) blackbody radiation from a given point on the surface of the white dwarf will vary as the "temperature wave" propagates around the star, with virtually the same effect as rotating hot spots. The details of the interaction between the star and material in the disk could of course be much more complex in either model.

In the oblique magnetic rotator model, there is no particular significance to the relation between the temperature of the hot spots and the mean surface temperature of the white dwarf. Let us consider instead the nonradial oscillator for a moment and suppose that the inferred modulation of the helium ions does indeed result; as suggested above, from a modulation of the temperature over the surface of the white dwarf. It is then interesting to ask how big a temperature variation is implied by the 
size of the emission line pulsations which we observe. Let us assume that i) the photons which ionize He II to He III are produced as the high-energy tails of blackbody radiation, and ii) the number of $\lambda 4686$ photons at any instant is directly proportional to the number of ionizing photons from the white dwarf. Since the photoionization cross-section is strongly peaked at the threshold of $54 \mathrm{eV}$, it will suffice here to consider only photons of this energy $\left(\equiv h \nu_{0}\right)$. If $F_{\nu_{0}}(T)$ is the flux per unit frequency interval of photons with energies near $h \nu_{0}$ from a blackbody at temperature $T$, then we must have

$$
f=\frac{\partial F_{\nu_{0}}}{\partial T} \frac{\Delta T}{F_{\nu_{0}}}
$$

where $f$ is the fractional modulation of the emission line and $\pm \Delta T$ is the temperature variation over the surface of the white dwarf. The right-hand side of this equation is to be evaluated at $\bar{T}$, the average temperature of the surface. From the expression for the blackbody flux at a given frequency we find

$$
\frac{\partial F_{\nu_{0}}}{\partial T} \frac{1}{F_{\nu_{0}}}=\frac{h \nu_{0}}{k T^{2}}\left(1-e^{-h \nu_{0} / k T}\right)^{-1}
$$

In the limit $\exp \left(\mathrm{h} \nu_{\mathrm{o}} / \mathrm{kT}\right) \gg 1$ this can be approximated as $h \nu_{\mathrm{o}} / \mathrm{kT}^{2}$. Using this latter expression, the measured value $f \approx 0.02$, and Kraft's (1959) estimate of the mean white dwarf temperature, $\bar{T}=8 \times 10^{4} \mathrm{o}$, we find $\Delta T / \bar{T} \approx 0.003$. This number is surprisingly small, though in view of the assumptions involved, the estimate is rather crude. 


\section{Geometrical Considerations}

We shall now consider a simple geometr: al model, based on the oblique magnetic rotator model, which can account for the wavelength-dependent phase shift of Pig. IV-4a and which illustrates the connection between this effect and the eclipse-associated phase shift of WPHN. We shall assume here that, as suggested by Katz (1975) and by Rees (1974), the pulsations arise from a reflection (and reprocessing) of radiation from the white dwarf hot spots off the back inner edge of the accretion disk. Such spots presumably arise at the magnetic poles from the inflow of matter along magnetic field lines and should radiate in the far ultraviolet, based upon a consideration of the accretion rate as deduced from the observed spin-up (Bath, Evans, and Pringle, 1974). Note that the white dwarf, the particles in the disk, and the binary system as a whole all rotate in the same sense: That the white dwarf rotates in the same sense as the Keplerian orbits in the disk follows from the fact that the period is observed to decrease, presumably due to the accretion of angular momentum. That the disk rotates in the same sense as the hinary follows from the sign of the Coriolis force. We shall assume furthermore, for the sake of simplicity, that the white dwarf spin and angular momentum vectors are very nearly aligned; i.e., the white dwarf spin axis is approximately perpendicular to the plane of the disk. Such an alignment is not improbable if the accreted angular momentum is appreciable (c.f. Bath, Evans, and Pringle, 1974). The model is shown schematically in Fig. IV-5a. The sense of rotation of both the disk and white dwarf is counterclockwise. For purposes of illustration the UV radiation pattern of the white dwarf has been idealized to a narrow beam whose axis of symmetry is located perpen- 
dicular to the spin axis of the white dwarf and we may, for convenience, refer to this radiation as "beamed." In reality one would expect a dipole-like radiation pattern with the magnetic axis at an arbitrary non-zero angle to the spin axis. It will turn out, however, that we can still develop this mode1 without having to specify such details about the system.

Now it is clear, upon inspection of Fig. IV-5a, that as the beam from the white dwarf sweeps across the back inner edge of the disk, it first illuminates receding material, then material traveling perpendicular to the line of sight, and finally approaching material. Thus the redshifted photons will be advanced in phase; the blue-shifted photons, retarded. This accounts qualitatively for the observed phase shift across the He II emission line. Since there is no relation between wavelength and velocity in the continuum, however, this effect is not observable in the continuum, and the broadband pulsations observed at any single wavelength represent an average over the entire back edge of the disk. When the disk is seen out of eclipse the average continum phase is, by symmetry, simply the phase at the center of the back edge (the mean phase point of Fig. IV-5a). It is clear that the He II line center, produced by material traveling perpendicular to the line of sight, will therefore be in phase with the continuum pulsations.

We note here that the N III - C III emission near $\lambda 4640$ shows no indication of a wavelength-dependent phase shift despite the clear excess signal (see Table IV-1, pg. S3) evident in the wavelength interval $4620-4660 \AA$. The poorer statistics in this feature relative to the stronger line at $\lambda 4686$ are probably not adequate to explain the absence 
of this effect. However, since this feature is not a true line, but rather a blend of several lines of both N III and C III, ${ }^{6}$ there is no well-defined relation between wavelength and velocity for these photons. As a consequence, we cannot expect to see the wavelength-dependent phase shift (which depends critically upon the wavelength-velocity relation) across this feature.

We can see the connection between the wavelength-dependent phase shift and the eclipse-associated phase shift as follows. We noted above that out of eclipse the continuum phase is defined by the mean phase point, located at the center of the back edge of the disk in Fig. IV-5a. However, when the system begins to move into eclipse a new effect manifests itse if in the continuum: As the dark companion moves across the disk (from the left in Fig. IV-5b) it first obscures that portion of the disk edge which is retarded in phase. As a result the mean phase point moves in the direction of advanced phase (to the right in Fig. IV-5b). The phase of the broadband signal thus abruptly begins to advance and continues $t w$ do so until the eclipse is total and the pulsations disappear. Similarly, as the system emerges from eclipse, the retarded side of the disk edge is revealed first. The signal phase thus appears retarded, but it catches up to its expected value as the inner edge emerges and the mean phase point returns to the center.

Now it is clear that if the pulsations arose instead at the surface of the white dwarf, as in the model of Bath, Evans, and Pringle (1974), the sign of the wavelength-dependent phase shift would disagree with the 
observations (i.e. the phase would decrease with increasing wavelength) because the velocity of the visible surface of the white dwarf is reversed from the velocity of the inner edge of the disk (see Fig. IV-5a). An analogous situation obviously arises in the case of the eclipse-associated phase shift as well.

We can make the inner edge model quantitative by means of the discussion which follows. First, however, we note that the characteristic dimensions of the binary system are less than $10^{11} \mathrm{~cm}(\mathrm{Kraft}, 1959)$ and consequently we shall assume that any phase delays resulting from light travel times can be neglected to first order $\left(71\right.$ i ight seconds $=2.1 \times 10^{12}$ $\mathrm{cm})$. In addition, although the details of the way in which the disk edge is excited as a function of time will depend upon the shape of the illuminating beam, we can (by the linearity of the Fourier transform) still calculate the relevant properties of the $71 \mathrm{~s}$ Fourier component of the reprocessed radiation if we assume a simple beam whose intensity varies sinusoidally with azimuth.

Now let $\psi$ be the angle from the center of the back inner edge of the disk to an arbitrary point on this edge as measured from the white dwarf (see Fig. IV-5a) so that the line of sight of the observer is located in the $\psi=0$ plane. The angle $\psi$ is related to the wavelength, $\lambda$, of an emission line photon from the edge by:

$$
\psi(\lambda)=\sin ^{-1}\left(\frac{\lambda_{0}-\lambda}{\beta \lambda_{0}}\right)
$$

where $B$ is the magnitude of the velocity (divided by the speed of light). at the disk edge, and $\lambda_{0}$ is the wavelength at the emission line center. In a wavelength interval extending from $\lambda_{1}$ to $\lambda_{2}$, the amplitude and phase 
of the signal will be proportional to the magnitude and equal to the complex phase, respectively, of the complex number $I_{B}\left(\lambda_{1}, \lambda_{2}\right)$, defined by

$$
I_{B}\left(\lambda_{1}, \lambda_{2}\right)=\int_{\lambda_{1}}^{\lambda_{2}} e^{-i m \psi(\lambda)} w(\psi(\lambda))\left|\frac{d \psi}{d \lambda}\right| d \lambda
$$

where $m=1$ if the disk is illuminated by a single beam and $m=2$ if there are two identical beams diametrically opposed. Note that in the case $m=2$, the true rotation period of the star is $142 \mathrm{~s}$, twice that of the resulting pulsations, while for $m=1$ the rotation and pulsation periods are equal. The factor $w(\psi)$ in the integrand is an angular weighting function which represents the fall-off in emission line strength with angle due to variation in $\hat{n} \cdot \hat{e}$ (where $\hat{n}$ and $\hat{\mathrm{e}}$ are unit vectors normal to the emitting surface element and toward the observer, respectively) and possibly due to effects of the disk shape as well.

We have fitted the five phase points in Fig. IV-4a using Eq. (IV.6) with $w(\psi)=\cos \psi$ (the results are not very sensitive to the precise form of $w(\psi))$ and minimizing $\chi^{2}$ as a function of $\beta$. In order to take account of the resolution of the ITS, the integrand in this equation was first convolved with a Gaussian distribution with $\sigma=5 \AA$. For the case $m=1$ we obtain an acceptable fit $\left(X^{2}=5.7\right.$, four degrees of freedom) for a velocity of $900 \pm 500 \mathrm{~km} \mathrm{~s}^{-1}$. For the case $m=2$ we again obtain an acceptable fit $\left(X^{2}=1.7\right)$, this time for a velocity of $2000 \pm 300 \mathrm{~km} \mathrm{~s}^{-1}$. The $m=2$ fit gives a pulsation amplitude profile (corresponding to Fig. IV-4b) that is substantially broader than observed (this point will be discussed further below); the $m=1$ fit gives a reasonable profile. In the $m=2$ case there is a secondary minimum of $x^{2}\left(x^{2}=0.5\right)$ corresponding . 
to a velocity of $120_{-30}^{+40} \mathrm{kim} \mathrm{s}^{-1}$. Our present view is that this latter solution is essentially an accident and is not physically meaningful; in any event it results in an amplitude profile that is much too narrow. Higher resolution observations should clarify this particular issue.

For reference we write Kepler's law for the velocities in the disk as :

$$
v=3640\left(\frac{M / M_{\theta}}{r_{9}}\right)^{\frac{1}{2}} \mathrm{~km} \mathrm{~s}^{-1}
$$

where $M$ is the mass of the white dwarf and $r_{9}$ is the distance (in units of $10^{9} \mathrm{~cm}$ ) from the center of the white dwarf co an arbitrary point in the plane of the disk. The mass if the white dwarf has been estimated at $\sim 1.1 M_{\Theta}$ with a probable error of $\pm 30 \%$ (Robinson, 1976a), the inner and outer disk radii at $\sim 3 \times 10^{9} \mathrm{~cm}$ (Bath, Evans, and Pringle, 1974) and $\sim 25 \times 10^{9} \mathrm{cin}$ (Kraft, 1959), respectively; the estimates of both of these radii (particularly the former) are also subject to considerable uncertainty. According to these estimates, we have velocities of $2200 \mathrm{~km} \mathrm{~s}^{-1}$ and 760 $\mathrm{km} \mathrm{s} \mathrm{s}^{-1}$ at the inner and outer disk edges, respectively. The data are thus consistent with an origin near the inner edge of the disk for the wavelength-dependent phase shift of Fig. IV-4a.

We can make the discussion of the eclipse-associated phase shift quantitative as in the case of the wavelength-dependent phase shift. In exact analogy to the above, the broadband signal amplitude and phase during ingress are derivable from the integral

$$
I\left(\psi_{\max }\right)=\int_{-\pi / 2}^{\psi_{\max }} e^{-i m \psi} \omega(\psi) d \psi
$$


vilere the upper cutoff, $\psi_{\max } \leqslant \pi / 2$, results from the eclipsing limb of the companion (assumed to be a knife-edge) and $m$ and $w(\psi)$ are defined as before. The signal amplitude and phase during ingress are shown as a function of $-\sin \left(\psi_{\max }\right)$ for the case $m=2$ in Fig. IV-6; we have again used $w(\psi)=\cos \psi$ as the weighting function. Different choices for $w(\psi)$ affect the shapes of these curves somewhat, but not the overall qualitative features (except that the pulsations vanish out of eclipse for $w(\psi)=1$ ). Note that since the velocity components of the white dwarf and companion perpendicular to the line of sight are nearly constant during the eclipse, the horizontal scale in Fig. IV-6 is nearly, though not exactly, proportional to time. The behavior of these functions during egress is symmetrical, except of course the sign of the phase shift is reversed. For the case $m=1$ the two curves are similar, the major difference being that i) the amplitude does not rise at first but is monotonically decreasing, and $i$ i) the phase shift only goes to $90^{\circ}$, not $180^{\circ}$. Unfortunately the interpretation of the effect is complicated in practice by the fact that as the eclipse proceeds the signal becomes weak and the phase consequently is difficult to determine. As a result, the original data of WPHN are insufficient to determine the value of $m$ on the basis of this effect. More recent data (Patterson et al., 1977) are similar1y ambiguous.

Despite the simplicity of the inner edge model and its quantitative success within the context of the wavelength-dependent phase shift, there are several reasons for believing that the pulsating region actually extends over the entire surface of the disk. In this model (based on a suggestion by Herbst; Hesser, and Ostriker [1974] and discussed below) the pulsations from the back half of the disk surface (the half further 
from the observer) dominate over those from the front ha1f. There need not actually be a (detectable) inner edge to the disk, but the net effect of this proposed front/back surface asymmetry is at least qualitatively the same as if the pulsations did originate at an inner disk edge.

The arguments in favor of pulsations from the disk surface are as follows:

i) The onset of the eclipse-associated phase shift (see Fig. 4 of WPHN) is roughly coincident with the onset of the eclipse itself (see Fig. 10 of Walker, 1956; Fig. 7 of Walker, 1958) at binary phase $\sim 0.9$. While the location of the inner edge is highly uncertain, it nonetheless seems unlikely that it is close enough to the outer edge to account for the phase shift beginning close to the time the dark companion first contacts the disk.

ii) Kraft's (1959) observation that the onset of rotational disturbance is also roughly coincident with the onset of eclipse, and Greenstein and Kraft's (1959) estimate of the size of the rotational broadening $\left(\sim 700 \mathrm{~km} \mathrm{~s}^{-1}\right)$ similarly indicate that at least the $\mathrm{DC}$ component of the $\lambda 4686$ emission extends over the entire disk.

iii) As noted above, the pulsation amplitude profile of the $\lambda 4686$ line (corresponding to Fig. IV-4b) predicted on the basis of the inner edge model (Eq. IV.6) and the best-fit values of $\mathrm{m}=2$ and $2000 \mathrm{~km} \mathrm{~s}^{-1}$ for the velocity, is substantially broader than observed. In other words, we detect too little signal in the shortest and longest wavelength bins of. Fig. IV-4b. One possible interpretation of this fact is that lower velocity, larger radius regions of the disk also contribute to the emission line pulsations, and consequently enhance the signal near the centra1 wavelengths of the 1 ine. 
Let us now consider the disk surface (ignoring the inner edge) A simple scale height argument shows that this surface will not be flat but rather slightly concave; the disk thickness should increase with increasing radius, $r$, as $r \%$ (Novikov and Thome, 1972). As a result of this curvature, all points on the disk surface can see the white dwarf, and can thus be excited by radiation originating at the surface of the dwarf. Furthermore, due to the combined effects of the disk shape and tilt (orbital inclination), the angle between the normal and the observer will vary over the surface so that the back half of the disk surface will be more visible than the front half. This can therefore provide the front/back asymmetry necessary for both the wavelength-dependent and eclipse-associated phase shifts. If we accept that the pulsations do indeed involve the disk surface, then we should try to understand the rather high velocities which result from the one-parameter fits (above). There are two possible explanations for this: i) The pulsed emission line contributions from the disk may be weighted toward the inner regions of the disk surface. ii) If there are substantial contributions from the front side of the disk surface, these will tend, in the m: 2 case, to diminish the magnitude of the wavelength-dependent phase shift, which in. turn will tend to raise the effective velocity as defined by Eqs. (IV.5) and (IV.6). In view of this 1atter possibility, one should perhaps not take too 1iterally the best-fit value of the velocity of $2000 \mathrm{~km} \mathrm{~s}^{-1}$ for the $\mathrm{m}=2$ case.

\section{Implications for Various : Models}

Let us summarize here the implications of our observations for the various models, other than the inner edge and surface models described 
above, which have been proposed to account for the optical pulsations in DQ Her. Recal1 that the observations of the eclipse-associated phase shift by WPHN provided the original argument against the pulsations being due to simple radial oscillations of the white dwarf. The wavelengthdependent phase shift described here clearly strengthens this case; only rotation or "pseudo-rotation" (non-radia1 oscillations) can produce the azimuthal variation $;$ phase $\mathrm{r}$ essary to explain both phase shifts. Similarly Lamb's $\quad ;$ explanation of the eclipse-associated phase shift as arising from arlitude modulation cannot account for the wavelengthdependent phase shift, and consequently also can be ruled out. (See the Appendix which follows for a further comment on Lamb's mode1.) The original model of WPHN, with the white dwarf oscillating in an $\ell=2$, $\mathrm{m}=0$ mode is likewise incapable of explaining the wavelength-dependent phase shift. The long-standing objection to the model of Bath, Evans, and Pringle (1974) - that it predicts the wrong sign for the eclipseassociated phase shift - is also strengthened by our observations. This nodel predicts the wrong sign for the wavelength-dependent phase shift as we11.

Our observations also have severa: interesting implications for the model of Nather and Robinson (1974), in which the pulsations originate at or near the outside of an optically thick photosphere suspended by Keplerian motion. The siga of the wavelength-dependent phase shift shows directly that the non-radial oscillations of the white dwarf, which are assumed to excite the pulsations in this model, must propagate around the star in the direction opposite the sense of rotation of the above Keplerian orbits. A similar conclusion can be drawn from the sign of 
the eclipse-associated phase shift. (These conclusions follow from the same argument used earlier to show that the model of Baths, Evans, and Pringle [1974] predicts the wrong sign for both phase shifts.) Although the Nather and Robinson model can thus account, in principle, for the sign of the lavelength-dependent phase shift, it is difficult to understand how the $\lambda 4686$ emission line, excited from below, can get out of the photosphere in this model. In addition, it is doubtful that this model can produc? sufficiently high Doppler velocities to account for the extent (in $\lambda$ ) of the emission line effect shown in Figs. IV-4a and $4 \mathrm{~b}$.' We note however that our resolution produces some additional broadening beyond that produced in the disk; higher resolution observations would be of interest in this regard.

We should point out here that, although we have the above objections to the specific non-radial oscillator model of Nather and Robinson, there is no reason fother than the often cited argument that only stellar rotation can provide the required frequency stability in DQ Her), that non-radial oscillations cannot replace the rotating hot spots in the inner edge and disk surface models which we have described above. Such non-radial oscillator models may in fact be appropriate in the case of UX Ursa Majoris. Nather and Robinson (1974) have observed an eclipseassociated phase shift in this object similar to the one in DQ Her but of opposite sign, and their data show in addition that the frequency stability of the pulsations in UX UMa is less than in DQ Her by 6 or 7 orders of magnitude.

Finally, we note that although the inner edge model which we have discussed in the preceding section is based in part upon the original 
suggestion by Katz (1975) that the pulsations originate at the inner edge of the accretion disk, Katz's interpretation of the eclipse-associated phase shift is quite different from the one we have presented above. In fact, our observations appear to rule out Katz's interpretation, as can be seen by the following argument: In Katz's picture, the eclipseassociated phase shift is due essentially to an interference between i) pulsations of angular frequency $w_{d}$ that are reprocessed from the inner edge of the disk, and $i i)$ pulsations of angular frequency $\omega_{c}$ that are reprocessed from matter corotating with the binary.

Since the white dwarf rotates in the same sense as does the binary system as a whole, it follows that $\omega_{d}-\omega_{c}=\Omega$, where $\Omega$ is the angular frequency of the orbital motion. In order that the interference effect between these two frequencies lead to a phase advance (a positive rather than a negative phase shift), Katz is forced to identify the dominant source of the observed pulsations (angular frequency $\omega_{0}$ ) with the lower frequency signal $\rightarrow$ the one criginating in the corotating matter, i.e. $w_{c}=w_{o}$. Consequently he predicts a weak, possibly not directly observable, signal at the higher frequency (the so-called upper orbital sideband) associated with the disk. In other words, if the angular frequency of the sideband is $\omega_{s}$, then $\omega_{s}=\omega_{d}=\omega_{0}+\Omega$. However, our observations show that the dominant signal is from the disk and thus $\omega_{d}=\omega_{0}$, contrary to Katz. ${ }^{7}$ A weak orbital sideband is still possible, but it must arise from the corotating matter and consequently be Zower in frequency than the observed signal: $\omega_{s}=\omega_{c}=\omega_{0}-\Omega$.

7 There is no evidence for a signal at $\omega_{0}+\Omega$ in our data, either in the broadband (for which we find a fractional mociulation of $0.12 \% \pm 0.12 \%$ ) or in the $\lambda 4686$ line (for which we find a fractional modulation of $0.2 \% \pm 0.4 \%$ ) . 
APPENDIX: PHASE SHIFT CAUSED BY FITTING A SINE

WAVE OF TIME-VARYING AMPLITUDE WITH A

CONSTANT AMPLITUDE SINE WAVE.

Lamb (1974), elaborating on a suggestion by WPHN, has noted that the systematic decrease in the pulsation amplitude of DQ Her during ingress (and similarly the systematic increase during egress) may lead to an apparent or artificial phase shift as the result of fitting the data to a constant amplitude sine wave. This raises the possibility (in Lamb's view) that the eclipse-associated phase shift in DQ Her may be due, at least in part, to an artifact of the data analysis procedures used by WPHN. However, we will show in this Appendix that this cannot be the case; the data reduction described by WPHN could not in fact have had any substantial effect on the measured phase.

In both of the above papers, the authors sketch an argument (based upon the idealized procedure of forcing the txtrema of the fitting function to coincide with the extrema of the observed light curve) which suggests that i) the sign of this artificial phase shift agrees with the sign of the eclipse-associated phase shift observed in DQ Her, and ii) the magnitude of the effect is large; i.e., apparent phase shifts of up to $90^{\circ}$ can occur. Here we treat the more realistic procedure (and the one actually used by WPHN to analyze their data) of fitting the observed light curve in a least squares sense, still to a constant amplitude sine wave, but using all points and not just those at the extrema. We find that artificial phase shifts still occur, but that i) the sign of the effect cannot be predicted in general, and ii) the magnitude of the effect is small, even for amplitudes which are relatively rapidly varying. 
Suppose that the signal we want to fit is given by

$$
f(t)=A(t) \cos \left(\omega t+\phi_{0}\right)
$$

where $A(t)$ is the time-varying amplitude and $\omega$, the angular frequency, is known. Let the duration of the observation be $T$ and let us require, for simplicity, that the observation spans an integral number, $n$, of cycles, i.e., $\omega T=2 \pi n$. We choose for our fitting function the constant amplitude sine wave:

$$
g(t)=a \cos (\omega t+\phi)
$$

and minimize the integral

$$
I(a, \phi)=\int_{0}^{T}[f(t)-g(t)]^{2} d t
$$

with respect to the two parameters $a$ and $\phi$. One can show that the phase which accomplishes this minimization (the measured phase) will be given by:

$$
\tan \phi=\frac{-\int_{0}^{T} A(t) \cos \left(\omega t+\phi_{0}\right) \sin \omega t d t}{\int_{0}^{T} A(t) \cos \left(\omega t+\phi_{0}\right) \cos \omega t d t}
$$

and the apparent phase shift $\delta$ is given by

$$
\delta=\phi-\phi_{0} \text {. }
$$

Now in the absence of a specific mode1 we cannot calculate $\delta$ explicit1y, bui se can still get an idea of the size of the apparent phase shift by assuming some representative forms for $A(t)$ :

i) Suppose that the amplitude decreases exponentially with time, i.e.

$$
A(t)=\alpha e^{-B t}, \quad B \geqslant 0 .
$$


Then we can show after some manipulation that

$$
-\frac{\beta}{2 \omega} \leqslant \tan \delta \leqslant \frac{\beta}{2 \omega}
$$

and $\tan \delta$ ranges over a11 values between these limits as $\phi_{0}$ ranges over a 11 angles.

ii) Suppose that the amplitude decreases linearly with time, i.e.,

$$
A(t)=\alpha(1-\beta t), \quad 0 \leqslant \beta^{\prime} T \leqslant 1 .
$$

We find:

$$
-\frac{\beta}{2 \omega} \gamma \leqslant \tan \delta \leqslant \frac{\beta}{2 \omega} \gamma
$$

where

$$
\gamma=\left[\left(1-\frac{\beta T}{2}\right)^{2}-\frac{\beta^{2}}{4 \omega^{2}}\right]^{-\frac{1}{2}}
$$

and again tand ranges over all values between these limits as $\phi_{0}$ ranges over all angles.

The above results show that the sign of the apparent phase shift depends on $\phi_{0}$, the true phase of the signal. Thus, contrary to the suggestion of WPHN and Lamb (1974), a decreasing amplitude does not always result in a positive apparent phase shift. Furthermore, the apparent phase shift is in general quite small: If the pulsation amplitude falls exponentially by a factor of $\leqslant e$ in one cycle, or linearly to zero in $\geqslant 2$ cycles; the above equations show that $|\tan \delta|<0.09$. Since the fitting intervals of WPHN were 5 cycles in length, any apparent phase shifts in their measurements on DQ Her must have been negligibly smal1. 


\section{REFERENCES}

Alme, M. L. and Wilson, J. R., 1974, Ap. J. 194, 147.

Anderson, L., 1977, submitted to Ap. J.

Avni, Y. and Bahca11, J. N., 1974, Ap. J. 191, 221.

Bahca11, J. N. and Bahcal1, N. A., 1972, Ap. J. (Letters) 178, L1.

Basko, M. M. and Sunyaev, R. A., 1973, Ap. E Space Sci. 23, 117.

Bach, G. T., Evans, W. D., and Pringle, J. E., 1974, MNRAS 166, 113.

Bopp, B. W., Grupsmith, G., McMi11an, R. S., Vanden Bout, P. A., and Wootten, H. A., 1973, Ap. J. (Letters) 186, L123.

Brigham, E. 0., 1974, The Fast Fourier Transform (Prentice Hall, Inc., Englewood Cliffs, N.J.).

Canizares, C. R. and McClintock, J. E., 1975, Ap. J. 200, 177.

Cooley, J. W. and Tukey, J. W., 1965, Math. of Computation 19, 297.

Crampton, D. and Hutchings, J. B., 1974, Ap. J. 191, 483.

Dahab, R. E., 1974, Ap. J. $187,351$.

Davidsen, A., Henry, J. P., Middleditch, J., and Smith, H. E., 1972, Ap. J. (Letters) 177, L97.

Davidsen, A., Margon, B., and Middleditch, J., 1975, Ap. J. 198 , 653. (DMM)

Deeter, J., Crosa, L., Gerend, D., and Boynton, P. E., 1976, Ap. J. 206, 861.

Dexsey, R., Bradt, H. V., Levine, A., Murthy, G. T., Rappaport, S., and Spada, G., 197.3, Ap. J. (Letters) 182, L25.

Forman, W. Jones, C. A., and Liller, W.;1972; Ap: J. (Letters) 177, L103. 
Giacconi, R., 1975, in Astrophysics and Gravitation, Proceedings, of the Sixteenth Annual Solvay Conference on Physics, The Solvay Foundation (L'Universite de Bruxe11es), p.27.

Giacconi, R., Gursky, H., Kellogg,.E.; Levinson, R., Schreier, E., and Tananbaum, H., 1973, Ap. J. 184, 227.

Greenstein, J. L. and Kraft, R. P., 1959, Ap. J. 130, 99.

Groth, E. J., 1974, Ap. J. 192, 517.

Herbst, W., Hesser, J. E., and Ostriker, J. P., 1974, AP. J. 193; 679.

Katz, J. I., 1975, Ap. J. 200, 298.

Kemp, J. C., Swed1und, J. B., and Wolsțencraft, R. D., 1974, Ap. J. (Letters) 193, L15.

Kraft, R. P., 1959, Ap. J. 130, 110.

Lamb, D. Q.) 1974, Ap. J . (Letters) 192, L129.

McClintock, J. E., Canizares, C. R., and Tarter, C. B., 1975, Ap. J. 198,641 .

Middleditch, J., 1975, Ph.D. Thesis, Lawrence Berkeley Laboratory Report LBL-3639.

Middleditch, J. and Ne1son, J., 1973, Ap. Letters 14, 129.

Middleditch, J. and NeIson, J., 1976, Ap. J. 208, 56̣7. (MN)

Moffett, T. J., Nather, R. E., and Vanden Bout, P. A., 1274, Ap. J. (Letters) 190, L63.

Nather, R. E. and Robinson, E. L.; 1974, Ap. J. 190, 637 .

Nather, R. E. and Warner, B.; 1969, MNRAS 143, 145.

Ne1son, J.E., Chanan, G. A., and Peterson, B., 1978, in preparation.

Novikov, I. D, and Thorne, K. S., 1972, in Black Holes, ed. C. Dewitt and B. S. Dewitt (Gordon \& Breach, New York); p.436. 
Patterson, J., Robinson, E. L., and Nather, R.E., 1977, preprint.

Prringle, J. E. and Rees, M. J., 1972, Astron. \& Astrophys. 21, 1 .

Rees, M. J., 1974, note added to Bath, Evans, and Pringle (1974), p.120.

Robinson, E. L., 1976a, Ap. J. 203, 485.

Robinson, E. L., 1976b, Ann. Rev. Astron. Ap. 14, 119.

Robinson, L. B. and Wampler, E. J., 1972, Pub. A.S.P. 84, 161.

Seaton, M. J., 1968, Adv. Atomic \& Mo1. Phys. 4, 338.

Shuiman, S., Friedman, H., Fritz, G., Henry, R. C., and Yentis, D. J., 1975, Ap. J. (Letters) 199, L101.

Swed1und, J. B., Kemp, J. C., and Wolstencroft, R. D., 1974, Ap. J. (Letters) 193, L11.

Tananbaum, H., Gursky, H., Kellogg, E. M., Levinson, R., Schreier, E., and Giacconi, R., 1972, Ap. J. (Letters) 174, 143.

Tarter, C. B. and McKee, C. F., 1973, Ap. J. (Letters) 186, L63.

Walker, M. F., 1954, Pub. A.S.P. 66, 230.

Walker, M. F., 1956, Ap. J. $\underline{123}, 68$.

Walker, M. F., 1958, Ap. J. 127, 319.

Walker, M. F., 1961, Ap. J. 134, 171.

Warner, B., Peters, W. L.; Hubbard, W. B., and Nather, R. E., 1972, MNRAS 159, 321. (WPHN) 


\section{FIGURE CAPTIONS}

Fig. I-1. Block diagram of the image-tube scanner (ITS), reprinted from Robinson and Wampler (1972). The minor modifications necessary to use the ITS in a high time-resolution mode (described in text) have not been indicated.

Fig. III-1. Spectrum of HZ Her, integrated from binary phase 0.18 to 0.26 . Normalization is uncertain below $\lambda 4000$. Emission features of N III and He II, Balmer absorption lines, and $\mathrm{n}_{\mathrm{b}}$ : kky lines ( $\mathrm{I} \lambda 5577$ and several lines of $\mathrm{Hg} \mathrm{I}$ ) are indicated. For reference, shapes of blackbody curves in an appropriate temperature range (arbitrary normalizations) are also shown.

Fig. III-2. A portion of the power spectrum of $\mathrm{HZ}$ Her. The spike corresponding to the $1.2 \mathrm{~s}$ pulsations is indicated.

Fig. III-3. (a) Normalized Fourier coefficients (see text) at an arbitrary frequency for each of the 255 wavelength channels ( $10 \AA$ in width) for $\mathrm{HZ}$ Her. No signaI is present; scatter is due to statistics. The probability of a point falling within the inner circle is $\frac{1}{2}$ and in about half of such samples one point should fall outside the outer circle.

(b) Normalized coefficients at the HZ Her frequency, broadband pulse phase adjusted to be $0^{\circ}$. The distribution resembles that of (a) but shifted to the right, characteristic of a signa1 distributed uniformly throughout the spectrum.

(c) Same as (b) but the contribution of a $0.2 \%$ pulsed fraction has been subtracted from each coefficient. Comparison with (a) shows that essentially a11 of the signal can be accounted for in this manner.

(d) Same as (b) but showing only those coefficients corresponding to emission features discussed in the text: the He II line (which falls in the two indicated channels) and the N III 
complex (which falls in the remaining four). These coefficients do not have large components in the direction specified by the broadband pulse phase (positive real axis); corresponding features consequently do not contribute substantially to the observed pulsations.

Fig. IV-1. Spectrum of DQ Her, integrated over one binary cycle, excluding eclipse. Emission lines are identified; Greek letters indicate the Balmer series.

Fig. IV-2. A portion of the power spectrum of DQ Her. The spike corresponding to the $71 \mathrm{~s}$ pulsations is indicated. The evident lack of power at the lowest frequencies is due to the smoothing procedure described in text.

Fig. IV-3. (a) Normalized Fourier coefficients for 255 wavelength channels at an arbitrary frequency near that of DQ Her. The scatter is due to statistics. The small displacement of the centroid of the distribution along the positive real axis is due to systematic effects such as fluctuations in telescope guiding, etc.

(b) Same as (a) but at the frequency of DQ Her. Broadband pulse phase is adjusted to be $0^{\circ}$. The large displacement of the centroid of the distribution to the right shows that the pulsations are distributed throughout the spectrum.

(c) Coefficients for the 5 channels of He II $\lambda 4686$ at a frequency corresponding to a noise spike in the power spectrum. Coefficients have been corrected for contribution due to the underlying continuum. Numbers increase with increasing wavelength across the line. There is no evidence of excess scatter in the imaginary direction:

(d) Samie as (c) but at the frequency of DQ Her. Points show excess scatter in the imaginary direction, which is evidence of a phase variation across the emission line. 
Fig. IV-4. (a) The wavelength-dependent phase shift in DQ Her: Pulsation phase across He $\lambda 4686$, corrected for contribution due to the underlying continuum. Vertical arrow indicates true line center. Red-shifted photons lead the continuum (phase $0^{\circ}$ ), blue-shifted photons $\mathrm{lag}$ the continuum. Because of the rather low resolution of the ITS, one must use care in any quantitative analysis of this figure (see text).

(b) Pulsation amplitude across He II $\lambda 4686$ corrected as in (a) . Due to the phase shift across the line, these amplitudes are not restricted (as is usually the case) to the components in phase with the broadband signal. The enhancement of the pulsations at longer wavelengths within the line is not understood; there is no corresponding asymmetry in the DC line profile. As in (a), the wavelength resolution of the ITS complicates any quantitative analysis of this figure.

Fig. IV-5. (a) A schematic model for the origin of the wavelengthdependent phase shift in DQ Her. As the white dwarf rotates (in the same direction as the disk), radiation from the hot spots first excites the material in the disk traveling away from the observer, then perpendicular to the line of sight, finally towards the observer; red-shifted protons are advanced in phase, blue-shifted phitons are retarded. This effect does not show up in the continuum; continuum phase is defined by the mean phase point at the center of the back edge.

(b) A schematic model for the origin of the eclipse-associated phase shift in DQ Her. As the dark companion moves across the disk during ingress, it first obscures regions of retarded pulsation phase in the disk. Mean phase point moves to the right and the continuum pulsation phase advances. The opposite effect occurs during egress. In actuality, the phase begins to advance at about the time the dark companion first contacts the disk. 
Fig. IV-6. The eclipse-associated phase shift in DQ Her: Theoretical curves for pulsation amplitude and phase during ingress, according to Eq. (IV.8), with $m \approx 2$ and $w(\psi)=\cos \psi$. In the case $m=1$, the phase shift only goes to $90^{\circ}$. Horizontal scale is approximately proportional to time. Curves during egress are reversed left to right and have the opposite sign for the phase. 


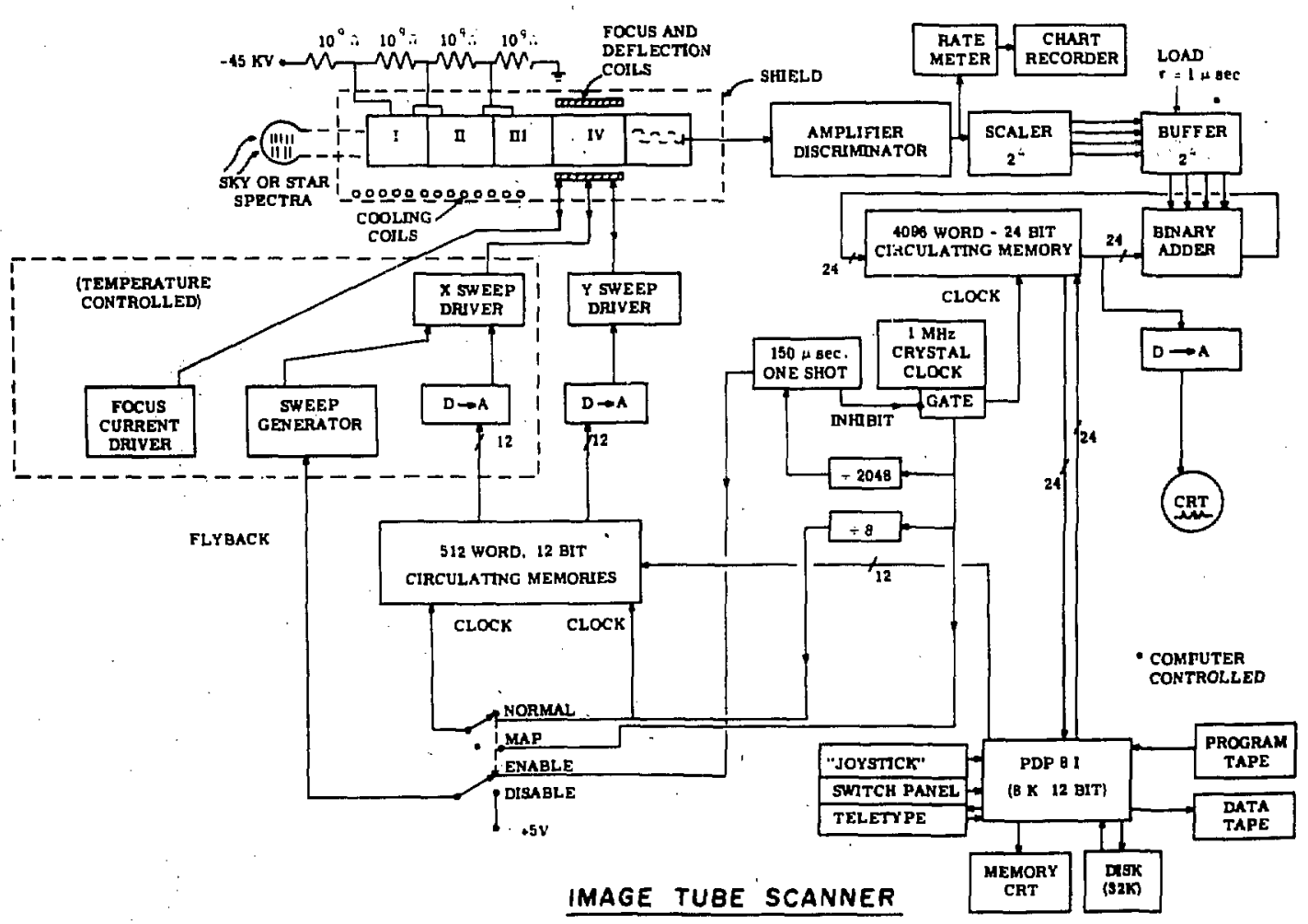

Blorik diagram of the scaunner system. 


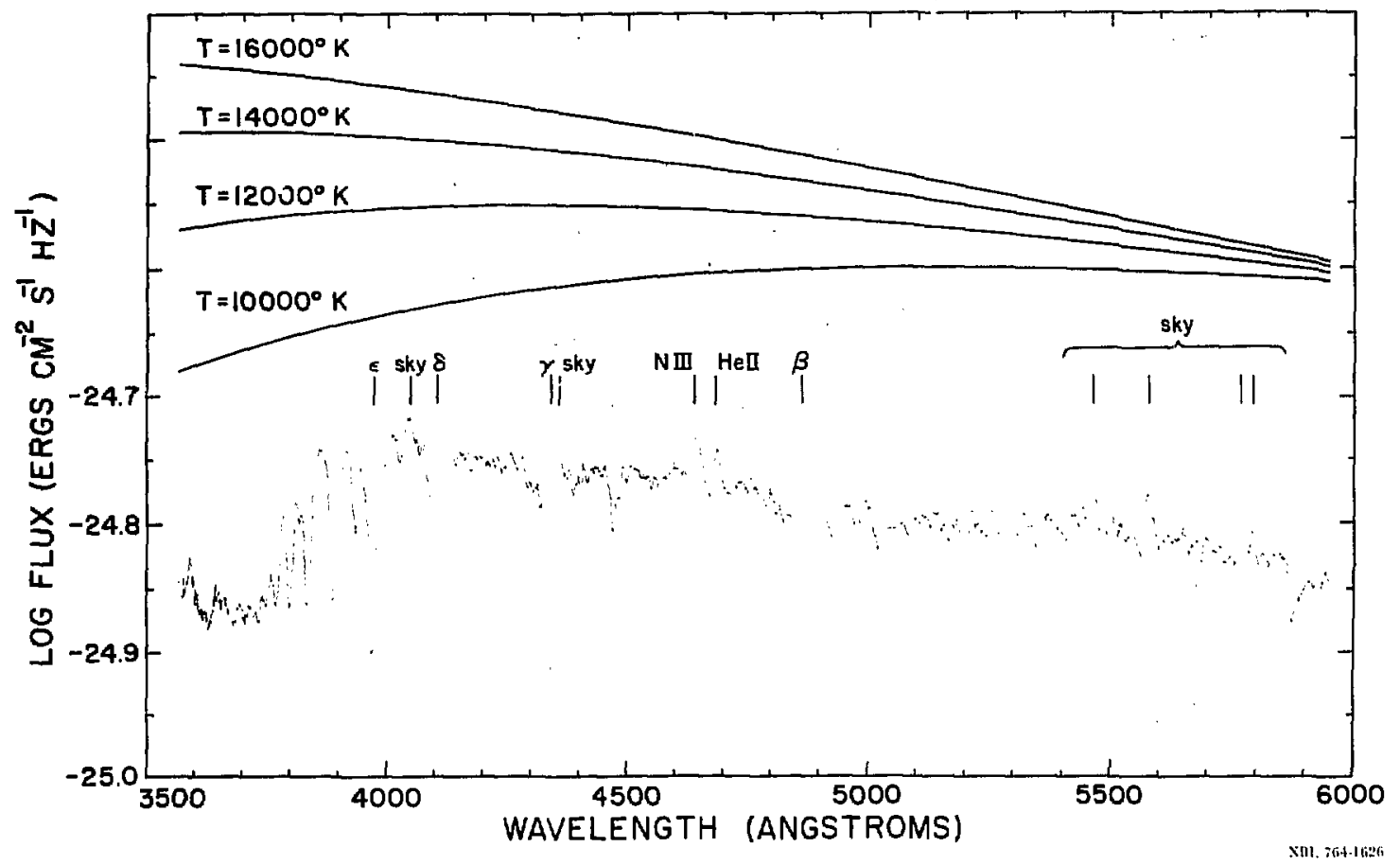

